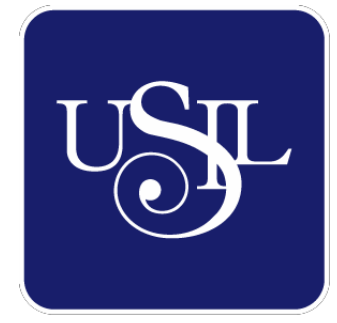

UNIVERSIDAD

SAN IGNACIO

FACULTAD DE HUMANIDADES

Carrera de Psicología

\title{
INFLUENCIA DE LA REGULACIÓN EMOCIONAL SOBRE LAS ESTRATEGIAS DE AFRONTAMIENTO AL ESTRÉS ANTE LA SITUACIÓN DE PANDEMIA EN UNIVERSITARIOS DE LIMA
}

Tesis para optar el Título Profesional de Licenciado en Psicología

\author{
DANIELA HUICHO BAUTISTA \\ (0000-0002-7060-4956)
}

\author{
Asesor: \\ Dr. Sergio Alexis Dominguez Lara \\ (0000-0002-2083-4278) \\ Lima - Perú \\ 2020
}




\section{Resumen}

La presente investigación tuvo como objetivo determinar si existe una influencia significativa de la regulación emocional sobre las estrategias de afrontamiento al estrés ante la situación de pandemia en universitarios de Lima. La muestra estuvo conformada por 333 estudiantes (74.8\% mujeres) con edades comprendidas entre los 18 y 29 años $(\mathrm{M}=22.2$; $\mathrm{DE}=2.94)$, los jóvenes se encontraban cursando entre el 3ro y 8vo ciclo de la universidad. Se utilizó el Cuestionario de Modos de Afrontamiento al Estrés (COPE) y el Cuestionario de Regulación Emocional (ERQ) ambos validados en Perú. Los resultados indican que la regulación emocional influye significativamente y en magnitud baja sobre alguna de las estrategias de afrontamiento al estrés. A pesar de realizar el estudio en un contexto diferente, debido al COVID-19, se obtuvo resultados similares respecto a algunas investigaciones anteriores.

Palabras clave: Estrategias de afrontamiento al estrés, regulación emocional, universitarios. 


\begin{abstract}
The objective of present research was to determine if there is a significant influence of emotional regulation on stress coping strategies in the face of the pandemic situation in young university students in Lima. The sample was made up of 333 students (24.9\% men and $74.8 \%$ women) aged between 18 and 29 years $(M=22.2 ; \mathrm{SD}=2.94)$, the young people were studying between the 3rd and 8th cycle of university. The Stress Coping Modes Questionnaire (COPE) and the Emotional Regulation Questionnaire (ERQ), both validated in Peru, were used. The results indicate that emotional regulation has a significant and low influence on some of the stress coping strategies. Despite conducting the study in a different context due to COVID-19, similar results were obtained with some previous research.
\end{abstract}

Keywords: Stress coping strategies, emotional regulation, university students. 


\section{Introducción}

En la actualidad, tanto el Perú como otros países del mundo se encuentran pasando una situación difícil debido a la pandemia del COVID-19. A partir de ello, las personas han empezado a considerar esta situación como estresante y amenazante, ya sea por el aislamiento social, las limitaciones en distintas actividades que antes se podían realizar, las consecuencias económicas, entre otros; provocando de esta forma un impacto psicológico significativo en la sociedad (Lorenzo, Díaz \& Zaldívar, 2020). En países como China se encontró que las personas tenían síntomas moderados a severos de depresión (16.5\%), ansiedad (28.8\%), y estrés (8.1\%) producto de la pandemia (Wang et al., 2020). A nivel nacional, con base en un estudio realizado por el Ministerio de Salud (MINSA) y la Organización Panamericana de la Salud (OPS, 2020), se encontró en una muestra de 57250 personas, sintomatología y problemas en lo que se refiere a salud mental, entre ellos ansiedad $27.85 \%$, depresión $12.37 \%$ y problemas de adicciones $5.18 \%$.

En el ámbito académico, antes del inicio de la pandemia, el proceso de aprendizaje venía acompañado con una experiencia de estrés y el uso de recursos para manejarlo dependía de las características del individuo (De la Fuente et al., 2020); el estudiante podía experimentar emociones negativas, falta de control, poca productividad, falta de concentración y fracaso académico, provocando que los jóvenes lidiaran con esta situación a partir de los recursos o mecanismos con los que contaban para adaptarse (García, Pérez-González, Pérez-Blasco \& Natividad, 2012).

Ligado a lo anterior, recientemente, los jóvenes se han visto aún más afectos debido al aislamiento social y el cambio de la modalidad de enseñanza: se pasó de llevar clases presenciales a recibir una educación a distancia con lo cual algunos jóvenes llegaron a sentirse saturados o sobrecargados, produciendo frustración al recibir mucha información a través de las plataformas educativas o tener problemas con la conectividad. Asimismo, se encontró un bajo nivel de competencia de autorregulación y disciplina; al pasar por todos estos cambios, los estudiantes han tenido que realizar reajustes que les permitan sobrellevar la situación, lo cual tiene una repercusión en el equilibrio socioemocional; se encontró que un $75 \%$ de los estudiantes habían experimentado ansiedad y depresión debido a las clases en la situación de pandemia (Pedró, 2020). 
Es importante mencionar que la vivencia que puedan tener los jóvenes ante la situación de pandemia puede variar de un país a otro, según una investigación realizada en España, los jóvenes entre las edades de 18 a 34 años debido al aislamiento social presentan un aumento en las dificultades para la concentración (53\%), evitan hablar o pensar en los problemas (37\%), presentan sentimientos depresivos o pesimistas (49\%), cambios de humor (56\%), dificultad en la capacidad de toma de decisiones y resolver problemas (35\%), pérdida del sentimiento de vitalidad y energía (53\%), así como irritación o enfado (58\%) (Balluerka, et al., 2020).

De igual forma, en otro estudio se encontró que la población más joven, es decir, personas entre las edades de 18 a 25 años presentan mayor sintomatología de estrés, ansiedad y depresión, relacionando estos aspectos a la necesidad de los estudiantes de adaptarse al nuevo contexto educativo por las clases virtuales, debido a ello se considera importante poner en marcha estrategias tempranas para la prevención y tratamiento de los efectos psicológicos que puede ocasionar la pandemia (Ozamiz, Dosil, Picaza \& Idoiaga, 2020).

Asimismo, el malestar emocional producto de la sobrecarga por la situación del aislamiento social, ha obligado a las personas a buscar soluciones ante estos momentos difíciles, lo cual puede producir sentimiento de incapacidad para hacer frente a los desafíos y a su vez sentimientos de soledad al no recibir el apoyo de otras personas que son significativas para el individuo; a partir de lo cual, se producirán desafíos que la sociedad tiene que hacer frente para salir adelante, debido a ello, las estrategias de afrontamiento al estrés toman un papel importante (Weide et al, 2020). Para la presente investigación, se considera pertinente definir el afrontamiento como un proceso cognitivo y conductual que va en constante cambio y que va permitir hacer frente a las demandas tanto internas como ambientales que son evaluadas como desbordantes de los recursos con los que cuenta una persona (Lazarus \& Folkman, 1984).

Con base en lo explicado anteriormente, a continuación, se profundizará en lo concerniente al afrontamiento. Este constructo está enfocado tanto en estilos como en estrategias. La primera de ellas definida como predisposiciones para lidiar con las situaciones o eventos estresores, los cuales determinarán las estrategias a utilizar, así como su estabilidad temporal y situacional (Solís \& Vidal, 2006), también son considerados como formas en las que la persona afronta el estrés. Mientras que las estrategias de afrontamiento son descritas como 
procesos más concretos y específicos, ya que dependerán del evento que se presente y sus efectos en el ambiente (Lazarus \& Folkman, 1984).

Ligado a lo anterior, entre los principales estilos de afrontamiento se encuentran los centrados en el problema, centrados en la emoción y el afrontamiento evitativo; el primero de ellos se refiere a la resolución de los problemas al manejar las demandas internas o externas que representan una amenaza o que desequilibran la relación que tiene un individuo con su entorno, mediante la modificación de las circunstancias problemáticas o la utilización de recursos que disminuyan las consecuencias de la situación ambiental (Lazarus \& Folkman, 1986).

Dentro del estilo de afrontamiento centrado en el problema, Carver, Scheier y Weintraub (1989), indican que se encuentran estrategias como el afrontamiento activo, proceso en el que la persona realiza acciones para evitar, apartar o disminuir las consecuencias negativas; otra estrategia es la planificación, en ella se realiza una planeación mental, para encontrar formas de acción, pasos a seguir y maneras de lidiar con el evento estresor; asimismo, la supresión de otras actividades permite que la persona se concentre en realizar los esfuerzos necesarios para afrontar una situación; la estrategia de contención de afrontamiento, va dirigido a actuar en el momento preciso y apropiado ante el evento estresor; por último, se encuentra la búsqueda de apoyo instrumental, esta estrategia permite encontrar recursos materiales en otras personas, ya sea consejos o información para poder manejar el problema.

Por otra parte, el estilo centrado en la emoción indica que las emociones tienen una función que permite la regulación emocional (RE), con lo cual se puede modificar y manejar el malestar y los estados emociones producto de eventos estresores, ya sea evitándola, realizando una reevaluación cognitiva o enfocando la atención en aspectos positivos del entorno o uno mismo (Di-Colloredo, Aparicio \& Moreno, 2006).

Entre las estrategias que conforman el estilo centrado en la emoción se encuentra la búsqueda de soporte emocional, la persona busca comprensión, apoyo y empatía para reducir el malestar emocional; luego está la estrategia de reinterpretación positiva y desarrollo personal, en ella la persona evalúa el problema atribuyéndole algo positivo o interpretándolo como un aprendizaje; en otro aspecto se encuentra la aceptación, esta estrategia permite realizar una evaluación primaria, en la que se es consciente del evento estresor y una fase de evaluación secundaria, en la que se acepta el convivir con la situación; la estrategia de enfocar y liberar 
emociones, se refiere a enfocarse en las consecuencias emocionales negativas expresándolas de forma abierta; finalmente, la estrategia acudir a la religión, permite disminuir la tensión por el evento estresor a partir de las creencias religiosas (Carver et al., 1989).

En lo que respecta al estilo de afrontamiento evitativo, este indica que ante un evento estresor la persona se desentiende de sus cogniciones y conductas, debido a ello, no realiza esfuerzos por controlar el problema, por el contrario, dispersa sus pensamientos realizando otras actividades, pero sin manejar la dificultad (Pérez, Morales \& Wetzaell, 2002). En este caso, dentro del estilo evitativo, Carver et al. (1989) mencionan que existen algunas estrategias, la primera, es la estrategia de desentendimiento conductual, en ella la persona abandona todos los objetivos que se han visto obstruidos por el evento estresor; por otro lado, se encuentra, el uso de sustancias, en el que la persona consume alcohol o drogas para evitar una situación estresante; asimismo, el uso del humor, estaría ligado con burlarse del evento estresor con la finalidad de evitar afrontarlo; la negación, por otro lado, se refiere a no creer que la situación que produce estrés existe, en otras palabras, se actúa y piensa como si este no existiera; por último, el desentendimiento mental son las actividades que una persona hace para mantenerse distraído de los pensamientos que le produce algún problema.

A partir de lo indicado anteriormente, algunas investigaciones realizadas en estudiantes universitarios demuestran que el afrontamiento se relaciona con variables como la ansiedad. Casari, Anglada y Daher (2014) desarrollaron una investigación con el objetivo de evaluar las diferencias y el grado de relación entre las variables de estrategias de afrontamiento y ansiedad ante exámenes, los resultados indicaron que ante niveles altos de ansiedad se incrementan las estrategias de evitación cognitiva, de aceptación - resignación, así como de evitación conductual. Por otro lado, en un estudio que quería conocer la relación entre los estilos de afrontamiento y la felicidad en estudiantes de una universidad particular de Lima se pudo encontrar que la felicidad se ha visto relacionada de forma inversa con las estrategias de desentendimiento mental y desentendimiento conductual (Córdova \& Sulca, 2018). De igual forma, Matalinares et al. (2016) al realizar una investigación con la finalidad de establecer la relación entre los modos de afrontamiento al estrés y el bienestar psicológico, encontraron que las estrategias de afrontamiento centradas en el problema y centrados en la emoción generan predisposición a mantener un bienestar psicológico. En el contexto del COVID-19, se encontró 
que las dimensiones de la respuesta emociones, es decir, la tristeza, la ansiedad, el miedo y el enojo estaban relacionadas de forma inversa con las estrategias enfocadas en el problema y las enfocadas en la emoción (Huang, Xu \& Liu, 2020).

Por otra parte, el modelo que plantea Lazarus y Folkman (1986) menciona que, si las demandas del ambiente son irrelevantes para la persona, no van afectar en ellas, debido a ello, no representará un compromiso en el ámbito físico, personal o social; pero si es relevante, va representar un compromiso ya que se dará un desequilibrio en el individuo, lo cual producirá una situación valorada como estresante y desencadenará un proceso en el que dará una evaluación y preparación para dar una respuesta de adaptación. Dicho proceso para evaluar la situación estresante se divide en: primaria y secundaria, la primera de ellas se refiere a la valoración que la persona hace de la situación, si significa algún riesgo, compromete su bienestar o supervivencia; por otro lado, en la secundaria se hace una evaluación de los recursos con los que cuenta la persona ya sean personales, sociales, económicos e institucionales, para hacer frente al evento estresor. Una vez realizada la evaluación la persona puede valorar la situación como: un desafío o una amenaza; en el primero el individuo considera que sus recursos son suficientes para enfrentar el evento estresor, promoviendo sentimientos de eficacia y logro; en lo que se refiere a la amenaza, la valoración es de no poseer recursos, debido a ello, su afrontamiento al estrés se vuelve poco eficaz y se presenta una percepción de ser incapaz de controlar la situación, de igual forma, se puede dar hasta respuestas ansiosas o depresivas.

Asimismo, existen recursos de afrontamiento al estrés con los que cuentan las personas, capacidades tanto internas como externas, para hacer frente a las demandas del evento estresor. El primero de ellos son los recursos físicos o biológicos, estos son todos los elementos que se encuentran disponibles en el entorno de un individuo. También se encuentran los recursos psicológicos o psicosociales, en ellos se consideran la capacidad intelectual, la autoestima, independencia, creencias y sentido de control de la persona. En los recursos culturales, están las normas, valores, creencias que se forman por la cultura de cada persona. Finalmente, en los recursos sociales se hallan sus sistemas de apoyo social, de los cuales se obtiene información, asistencia o soporte emocional para afrontar aquellas situaciones de estrés (Lévano, 2003).

De igual forma, se considera que las diferencias individuales pueden influir en el afrontamiento al estrés de dos formas, el primero de ellos indica que la persona adquiere un 
conjunto de estrategias de carácter estable, es decir, un afrontamiento disposicional que será estable a través del tiempo y en las situaciones que se presenten, asimismo, se explica que los rasgos de personalidad van a predisponer al individuo para usar ciertas estrategias para afrontar un evento estresor. Por el otro lado, se menciona un modelo de carácter situacional, eso quiere decir que el afrontamiento es cambiante y dinámico, en ella se hace uso de varias estrategias sin depender de las características de personalidad (Lazarus \& Folkman, 1984).

Como se indicó anteriormente, se puede observar que la situación por la COVID-19 ha generado una repercusión en la salud mental, con lo cual las personas manifiestan emociones más intensas y de una duración prolongada que lleva al individuo a encontrar maneras de regularse para disminuir el malestar o modular las consecuencias producto del estrés (Muñoz et al., 2020). En el caso de los jóvenes, ellos tendrán que adaptarse a las diversas emociones negativas y positivas que van a experimentar ante las demandas del contexto educativo (Chau \& Saravia, 2014). Es en este punto donde la RE cumple un papel importante dado que ha sido vinculada con el afrontamiento al estrés.

A partir de lo indicado, la RE se refiere al esfuerzo para cambiar cómo se siente uno mismo mediante el control de los elementos que están produciendo la experiencia emocional (Gross \& Thompson, 2009). Asimismo, la RE está representada por dos dimensiones: la reevaluación cognitiva y la supresión. La primera hace referencia a modificar el proceso en el que surge la emoción para conseguir que el impacto que tenga sobre una persona sea diferente. Por otra parte, en la supresión se inhibe la expresión de una respuesta emocional modificando la conducta del individuo más no el impacto o efecto de la emoción sobre la persona. En este caso, este tipo de RE está enfocado en la respuesta; es decir, se presenta después del proceso que genera la emoción (Gross \& John, 2003).

Es importante mencionar que existen algunas consecuencias derivadas de la supresión ya sea en la experiencia afectiva, fisiológica, la memoria o los procesos interpersonales. Silva (2005) indica que el uso de la supresión disminuye la expresión afectiva lo cual produce una menor comunicación del estado en el que se encuentra la persona. De igual forma, se tiene una mayor activación del sistema simpático, se da un mayor esfuerzo cognitivo en el proceso emocional y se produce efectos negativos hacia las demás personas. Ligado a lo anterior, se puede inferir que ante la situación de pandemia los jóvenes pueden experimentar una mayor 
supresión emocional lo cual podría suponer una menor comunicación de sus sentimientos con su entorno lo que podría interferir en su desempeño académico. Gross y John (2003) indican que personas que utilizan con mayor frecuencia la supresión son menos conscientes de sus propios sentimientos por lo que presentan problemas en sus relaciones sociales, en consecuencia, existe una resistencia a buscar o recibir ayuda por lo que se suele tener menos apoyo social y habilidades de afrontamiento deficientes.

Por el contrario, la reevaluación cognitiva, permite tener una experiencia menos desagradable frente a estímulos aversivos, así como una activación fisiológica menor. Por otro lado, debido a que la reevaluación no requiere esfuerzos de autorregulación, no interferirá en los recursos cognitivos de la persona por lo que no afectará funciones como la memoria (Silva, 2005). Por tal motivo, los estudiantes que utilicen la reevaluación cognitiva para regular sus emociones podrían experimentar la situación de forma menos aversiva por lo que no se vería afectado su desempeño académico. Por otro lado, Gross y John (2003) consideran que la reevaluación cognitiva es un método efectivo para regular las emociones porque requiere menos recursos cognitivos y fisiológicos comparado con la supresión. Asimismo, debido a que la reevaluación cognitiva ocurre antes de la completa activación de la respuesta emocional, la RE no crea una discrepancia entre la experiencia interna y la expresión externa; personas que utilizan esta forma de regulación suelen compartir más sus emociones, mantener relaciones cercanas con amigos; asimismo, el afrontamiento al estrés se puede ver asociado debido a que maximiza la experiencia de emociones positivas, reduciendo el impacto de las emociones negativas y permitiendo resultados más adaptativos (Gross \& John, 2003).

De igual forma, se puede encontrar que una RE adaptativa implica tres factores importantes, la primera es la conciencia o conocimiento sobre las emociones, ya que permite mejorar las estrategias que ayudan al individuo a regularse. El segundo factor es el objetivo de la RE, es decir, lo que uno quiere lograr, que permite un aumento o disminución de la expresión, experiencia o fisiología de las emociones. El último factor son las estrategias, mediante el cual la persona logrará los objetivos para regular sus emociones (Feldman, Gross, Conner \& Benvenuto, 2001).

Otro aspecto relevante dentro de esta variable es el modelo modal de la RE propuesto por Gross (1999). Este modelo indica que la RE se puede estudiar a partir de las estrategias que 
se utilicen dentro del proceso en el que se genera una emoción, en dicho proceso existen distintas fases: situación, atención, interpretación y respuesta. En cada una de ellas se hará uso de estrategias diferentes, las cuales están divididas en dos grupos más grandes la regulación centrada en los antecedentes de la emoción y la regulación centrada en la respuesta emocional.

Como se indicó anteriormente, existen cuatro fases principales y dentro de ellas distintas estrategias, la primera llamada la selección de la situación, sucede antes de la primera fase del proceso, en ella se explica que la persona puede decidir a qué situaciones se quiere exponer, por ejemplo, aquellas situaciones que generan emociones negativas producen un aprendizaje para que en un futuro se evite repetir aquellas emociones no deseadas; por lo que la persona preferirá situaciones en las que perciba experiencias positiva; esto se ve muy ligado con las estrategias de afrontamiento del estilo centrado en el problema, específicamente con el afrontamiento activo ya que en él se realizan acciones para reducir consecuencias negativas de un evento estresor (Carver et al., 1989). Dentro de la primera fase del modelo de Gross (1999) denominada situación, se encuentra la estrategia de modificación de la situación, cuando la persona ya está dentro de un suceso o evento, puede cambiar los elementos en la dirección que prefiera, modificando las acciones que tome u omita; esta fase se puede relacionar con la estrategia de afrontamiento de planificación debido a que en ella la persona intenta encontrar maneras para realizar acciones y lidiar con la situación estresante (Carver et al., 1989).

Dentro de la segunda fase llamada atención se encuentra la estrategia de modulación de la atención, una vez los componentes u aspectos de una situación se hayan establecido, se puede dirigir la atención a unos estímulos y no a otros, lo cual permitirá regular la respuesta emocional (Gross, 1999); este aspecto se puede ver relacionado con la estrategia de desentendimiento mental que se encuentra dentro del estilo evitativo, en ella se menciona que el individuo realizará actividades para mantenerse distraído de aquellos pensamiento que se producen por algún problema y de esta forma modular lo que siente; por otro lado, la persona puede enfocar su atención en aspectos como la religión que le permitirán disminuir el malestar producto del evento estresor (Carver et al., 1989). Para la fase de interpretación, se encuentra la estrategia de cambio cognitivo, la cual se refiere a como la persona va interpretar la situación, esto representa un mecanismo importante ya que modulará la reacción emocional Gross (1999); en este caso, el afrontamiento centrado en la emoción, específicamente, la estrategia de reinterpretación 
positiva estaría relacionada ya que mediante ella se evalúa el problema para atribuir algo positivo e interpretarlo como un aprendizaje, de igual forma, la estrategia de aceptación permitiría realizar evaluaciones para poder aceptar la situación y convivir con ella.

Dentro de la fase de respuesta, la estrategia de regulación de la respuesta emocional, hace referencia a que el individuo actúa frente a los componentes de la emoción que se activa para modificar la experiencia emocional, la manifestación de la conducta y la respuesta fisiológica (Gross \& Thompson, 2009); relacionando este aspecto con las estrategias de afrontamiento se puede encontrar que la búsqueda de soporte social por razones emocionales podría estar relacionada al igual que el afrontamiento religioso o la negación debido a que con ellas se podría modular la experiencia emocional (Carver et al., 1989).

Monteiro, Balogun y Oratile (2014) indican que ambos constructos tienen el objetivo de adaptar al individual a eventos estresores, en lo que respecta al afrontamiento, involucraría esfuerzos por regular emociones, pensamientos, comportamientos y reacciones físicas en respuesta a situaciones que considera estresantes; a pesar de ello, el afrontamiento se puede distinguir de la RE ya que va dirigida a reducir efectos negativos y por periodos más largos de tiempo; en el caso de la RE, las emociones se expresan y varían según la adaptabilidad y es necesario regularlo con frecuencia.

Según Ramón y Ramón (2017) una adecuada capacidad para modular las respuestas emociones, va a permitir satisfacer los objetivos individuales y, a su vez, las demandas de los eventos estresores. Es por ello que las emociones son un elemento importante para saber cómo la persona va percibir una situación estresante y cómo actuará ante ello. Por otro lado, Pascual y Conejero (2019) indican que el afrontamiento al estrés va a permitir que el individuo pueda librarse las experiencias emocionales que son consideradas desagradables. Asimismo, Souto (2013) considera que la RE es un componente importante debido a que permite moderar las emociones que proporcionarán mecanismos adaptativos, lo cual derivará en un mejor manejo de factores o eventos estresores en la vida de los estudiantes. Finalmente, Midkiff, Lindsey y Meadows (2018) indican que existe una relación entre ambas variables; en un estudio que realizaron se encontró que las estrategias de afrontamiento al estrés poco adaptativas, regulan las emociones de forma superficial, es decir no solucionan o alteran las emociones por el contrario impacta de forma negativa en la vida de la persona. 
De acuerdo a lo indicado anteriormente, a continuación, se abordará algunas investigaciones que guardan relación con el tema de investigación.

Ong y Thompson (2019) desarrollaron una investigación con el objetivo de explorar la relación entre la RE, el afrontamiento y la conducta suicida en estudiantes de Hong Kong. Los participantes fueron 117 universitarios, que se encontraban en un rango de edad de 18 a 28 años. Los instrumentos utilizados fueron el Cuestionario de Regulación emocional (ERQ; Gross \& John, 2003) que cuenta con 10 ítems divididos en dos dimensiones: la supresión y la reevaluación cognitiva. Asimismo, se aplicó el Cuestionario COPE Revisado (R-COPE; Zuckerman \& Gagne, 2003) conformada por 40 ítems divididos en cinco dimensiones: autoayuda, enfoque de afrontamiento, acomodación, evitación y autocastigo. Los resultados demuestran que existe una relación estadísticamente significativa y positiva entre la reevaluación cognitiva y las dimensiones de acomodación $(r=.26 ; p<.05)$, el enfoque de afrontamiento $(r=.23 ; p<.05)$ y autoayuda $(\mathrm{r}=.21 ; \mathrm{p}<.05)$ pertenecientes al estilo centrado en el problema y centrado en la emoción. Asimismo, existe una relación significativa y positiva entre la supresión y el afrontamiento evitativo $(r=.26 ; p<.05)$ y de autocastigo $(r=.24 ; p<$ $.05)$. Se concluye que la capacidad de regular emociones reinterpretando una situación o evento estresor es una forma beneficiosa de afrontamiento.

Piri, Amiri, Bazzazian y Ghamari (2019) realizaron un estudio con la finalidad de examinar el papel mediador de las estrategias de afrontamiento y su relación con las dificultades de la RE y la adicción al internet en estudiantes universitarios. La muestra estuvo conformada por 375 estudiantes universitarios (información sobre sexo y edad no especificada) y se usó el Inventario de Respuestas al Afrontamiento (Billings \& Moss, 1984) que consiste en 32 ítems divididos en: estilos basados en la aproximación al problema (análisis lógico, reevaluación positiva, búsqueda de guía y soporte y resolución de problemas) y estilos de evitación o centrados en la emoción (evitación cognitiva, aceptación-resignación, búsqueda de recompensas alternativas y descarga emocional). De igual forma, se hizo uso de la Escala de Dificultades en la Regulación Emocional (Gratz \& Roemer, 2004) que incluye 41 ítems divididos en las siguientes dimensiones: no aceptar la respuesta emocional, dificultad para participar en un comportamiento dirigido a objetivos, dificultades en el control de impulsos, falta de conciencia emocional, acceso limitado a estrategias de RE y falta de claridad emocional. Los resultados indicaron que existe una relación directa y significativa entre las dificultades en la RE y las 
estrategias de afrontamiento del estilo evitativo $(r=.527 ; p<.05)$; las dimensiones de no aceptar la respuesta emocional $(r=.480 ; p<.05)$, dificultad en el control de impulsos $(r=.430 ; p<$ $.05)$, y el acceso limitado a estrategias de $\operatorname{RE}(r=.463 ; p<.05)$ se relacionan significativamente con las estrategias del estilo centrado en la evitación o centrados en la emoción (evitación cognitiva, aceptación-resignación, búsqueda de recompensas alternativas y descarga emocional). Se concluye que las dificultades en la RE en estudiantes universitarios pueden tener un impacto positivo en las estrategias de afrontamiento de estilo evitativo.

Midkiff, Lindsey y Meadows (2018) plantearon un estudio con la finalidad de examinar el efecto mediador del afrontamiento de la autoeficacia en la relación entre la desregulación emocional y la frecuencia de autolesiones. Los participantes fueron 187 estudiantes universitarios, $76.5 \%$ mujeres y $23.5 \%$ hombres, sus edades oscilaban entre los 18 y 26 años. Se utilizó la Escala de afrontamiento de la Autoeficacia (Chesney, Neilands, Chambers, Taylor \& Folkman, 2006) que cuenta con 26 ítems divididos en tres subescalas: detener emociones y pensamientos desagradables, uso del afrontamiento centrado en el problema y obtener apoyo de familiares y amigo; de igual forma, se aplicó la Escala de Dificultades en la Regulación Emocional (Gratz \& Roemer, 2004) que incluye 41 ítems y seis dimensiones: no aceptar la respuesta emocional, dificultad para participar en un comportamiento dirigido a objetivos, dificultades en el control de impulsos, falta de conciencia emocional, acceso limitado a estrategias de RE y falta de claridad emocional. Los resultados indican que el afrontamiento de autoeficacia se relaciona negativamente con las dificultades de $\operatorname{RE}(r=-.710 ; p<.001)$, asimismo, se encontró que a medida que las personas tienen mayores dificultades para regular sus emociones se espera que tengan menor afrontamiento de autoeficacia $(\mathrm{F}(1.185)=188.14$, $\mathrm{p}<.001)$. Se concluye que el nivel de dificultad que tiene un individuo para regular sus emociones es un predictor de la confianza que tendrá para hacer frente a desafíos o amenazas.

Smrtnik y Prosen (2016) desarrollaron una investigación con el objetivo de conocer la asociación que existe entre el afrontamiento y las estrategias de RE considerando el género y nivel de educación. La muestra estuvo conformada por 194 adultos de los cuales el 57.22\% fueron hombres y el $42.78 \%$ mujeres; dichos participantes se encontraban entre las edades de 20 a 65 años; asimismo, se dividió en tres grupos según la edad, de 20 a 29 años $(30.41 \%$, M = $24.59, \mathrm{DE}=2.67)$, de 30 a 45 años $(27.84 \%, \mathrm{M}=38.43, \mathrm{DE}=4.40)$, y personas de 46 a 65 años (41.75\%, M = 50.44, DE= 4.13); de igual forma, se separó en tres grupos según su nivel de 
educación, primaria $(21,33 \%)$, secundaria $(23,20 \%)$ y universidad $(55,67 \%)$. Por otro lado, se utilizó el Cuestionario de Formas de Afrontamiento (Folkman \& Lazarus, 1986) conformado por 66 ítems que están divididos en ocho dimensiones: afrontamiento confrontativo, distracción, autocontrol, búsqueda de soporte social, aceptar la responsabilidad, evitación, resolución de problemas planificada y reevaluación positiva; asimismo, se aplicó el Cuestionario de Estrategias de Regulación Emocional (ERQS; Gross \& Thompson, 2009) que consta de 27 ítems divididos en cinco dimensiones: selección de la situación, modificación de la situación, modulación de la atención, cambio cognitivo y la regulación de la respuesta emocional. Los resultados indican que se encontraron correlaciones estadísticamente significativas entre la reevaluación cognitiva y las dimensiones de resolución de problemas planificada $(r=.31 ; p<$ .01) y la reevaluación positiva $(r=.28 ; p<.01)$; de igual forma entre la supresión y las dimensiones de distracción $(r=.24 ; p<.01)$ y autocontrol $(r=.21 ; p<.01)$. Se concluye que las correlaciones entre el afrontamiento y la RE en su mayoría no son significativas, debido a ello se considera que la asociación entre ambas variables es relativamente baja.

Monteiro, Balogun y Oratile (2014) plantearon un estudio con la finalidad de conocer la relación entre las estrategias de afrontamiento y RE. Los participantes fueron 128 estudiantes universitarios, 64 mujeres y 64 hombres, con edades que oscilaban entre los 18 y 29 años. Los instrumentos que se utilizaron fueron el Inventario de estrategias de afrontamiento (CSI; Tobin, Holroyd \& Reynolds, 1984) conformado por 72 ítems divididos en ocho escalas primarias (resolución de problemas, reestructuración cognitiva, soporte social, expresión emocional, evitación del problema, ilusión, retiro social, autocrítica) y cuatro escalas secundarias (compromiso centrado en el problema, compromiso enfocado en la emoción, desconexión centrada en el problema y desconexión centrada en la emoción). Asimismo, se usó la Escala de Dificultades en la Regulación Emocional (Gratz \& Roemer, 2004) que incluye 41 ítems divididos en 6 dimensiones: no aceptar la respuesta emocional, dificultad para participar en un comportamiento dirigido a objetivos, dificultades en el control de impulsos, falta de conciencia emocional, acceso limitado a estrategias de RE y falta de claridad emocional. Los resultados mostraron que la subescala de falta de conciencia emocional tiene una correlación positiva con la dimensión de desconexión enfocado en el problema $(r=.178 ; p<.01)$; asimismo, se encontró una relación significativa entre el acceso limitado a estrategias de RE y las dimensiones de desconexión centrado en el problema $(r=.260 ; p<.01)$ y la emoción $(r=.358 ; p<.01)$; por 
último, también la relación entre la falta de claridad emocional y la desconexión centrada en el problema $(r=.238 ; p<.01)$ y emoción $(r=.189 ; p<.01)$ son estadísticamente significativos. Se concluye que existen una relación significativa entre las dificultades en la RE y las estrategias de afrontamiento, por lo que dependiendo como la persona se regula, la emoción toma un papel importante en como afrontaran el estrés. A pesar de esto, la relación es compleja porque no todas las dimensiones de las dificultades de la RE han sido correlacionadas con las estrategias de afrontamiento.

Wong et al. (2013) desarrollaron un estudio con la finalidad de examinar como el afrontamiento y la RE son capaces de diferenciar el inicio de prescripciones médicas y la magnitud del reciente uso. Para ello, los participantes estuvieron conformados por 560 jóvenes entre las edades de 16 a 25 años que hubieran usado indebidamente medicamentos recetados en los últimos 90 días. Los instrumentos fueron el Cuestionario Breve del COPE (Carver, 1997) que consta de 24 ítems y siete subescalas: autodistracción, afrontamiento activo, búsqueda de soporte emocional, búsqueda de soporte instrumental, desfogue, replanteamiento positivo y planificación. Asimismo, se usó el Cuestionario de regulación emocional (Gross \& John, 2003) que contiene 10 ítems dividido en dos dimensiones: la supresión y la reevaluación positiva. Los resultados indican que existen 4 clases o perfiles según las estrategias de afrontamiento y ER, la primera es la clase 1 denominado "supresores" en el que se encuentra el 15\% de los participantes, ellos se caracterizan por usar más la supresión. La clase 2 denominada dependientes de otros, abarca el $27 \%$ de los participantes y se caracterizan por utilizar las estrategias de búsqueda de apoyo instrumental. Por otro lado, la clase 3 llamados afrontadores autosuficientes está conformada por el $27 \%$ de los participantes, ellos cuentan con un bajo respaldo en la búsqueda de soporte emocional e instrumental. Por último, la clase 4 denominada afrontadores activos consta del $30 \%$ de los participantes, ellos utilizan en mayor medida el afrontamiento, la búsqueda de apoyo emocional e instrumental, el replanteamiento positivo y la planificación. Se concluye que en la clase 1 la dimensión de supresión no estaría relacionada con alguna estrategia de afrontamiento, para la clase 2 la búsqueda de soporte social tampoco estaría asociada con alguna dimensión de la RE, asimismo, en la clase 3 las estrategias de afrontamiento no estarían asociadas con la RE, a pesar de ello, en la clase 4 si existiría una relación significativa entre las estrategias de afrontamiento y la dimensión de reevaluación cognitiva. 
Cabral, Matos, Beyers y Soenens (2012) realizaron una investigación con el objetivo de examinar las interrelaciones entre las medidas de apego parental, los procesos de RE y las estrategias de afrontamiento. La muestra estuvo compuesta por 942 estudiantes universitarios de primer año, $36.5 \%$ hombres y $63.5 \%$ mujeres, que se encontraban en un rango de edad entre los 17 a 20 años. Se aplicó el Inventario de COPE (Carver, Scheier, \& Weintraub, 1989) que incluye 15 ítems que se dividen en cuatro dimensiones: Activo/ reflexivo, búsqueda de apoyo, evitación y replanteamiento positivo. De igual forma, se utilizó el Trait Meta-Mood Scale (TMMS; Salovey et al., 1995) que consta en 21 ítems que se dividen en cuatro dimensiones: supresión, claridad, regulación y dificultades en la represión defensiva. Los resultados indican que la supresión se relaciona positivamente con el afrontamiento evitativo $(r=.27 ; p<.05)$ y el replanteamiento positivo $(r=.12 ; \mathrm{p}<.05)$; por otro lado, la regulación se relaciona positivamente con el afrontamiento activo $(r=.34 ; p<.05)$, la búsqueda de apoyo $(r=.32 ; p<$ $.05)$ y el replanteamiento positivo $(r=.54 ; p<.05)$. Se concluye que existe una correlación de baja magnitud entre los constructos de afrontamiento y RE.

De acuerdo con las investigaciones señaladas, el presente estudio se justifica a nivel teórico, debido a que a pesar de que existen investigaciones sobre las estrategias de afrontamiento y RE en universitarios; son muy escasas las que relacionan dichas variables en el Perú y Latinoamérica, ya que solo se encontró estudios que abarcan una variable, o relacionan una de ellas con otros constructos diferentes a las del presente estudio. Por otro lado, es importante mencionar que en el contexto de la pandemia del COVID-19, se ha visto un incremento de problemas de salud mental lo cual puede llegar a repercutir en como un individuo va gestionar sus emociones y afrontar una situación que excede sus recursos. Debido a ello, se considera que esta investigación es de importancia teórica ya que servirá para conocer la influencia existente de la RE sobre las estrategias de afrontamiento ante la situación de pandemia en universitarios de Lima.

Asimismo, a nivel práctico, se justifica porque mediante la evaluación de las estrategias de afrontamiento y RE en universitarios, se puede brindar información que permita concientizar a las personas sobre el impacto que ocasionar la situación de pandemia en la vida cotidiana de los jóvenes y de este modo se tome acciones en caso exista un impacto negativo en la persona; autores recomiendan la implementación de programas de intervención sobre afrontamiento que promuevan el uso de estrategias que le permita al individuo hacer frente a eventos o situaciones 
estresantes y de esta forma generar bienestar en ellos; así como programas vivenciales en el que tengan un espacio para expresar sus emociones, que les brinde soporte y puedan fomentar habilidades de autorregulación (Gaeta \& Martín, 2009; Urbano, 2019; Gutiérrez, 2018); por lo cual, estos puntos se consideran importante de implementar a partir del presente estudio. Por otro lado, se espera que en futuras investigaciones puedan tomar en cuenta los resultados obtenidos y quizás abarcar otros aspectos que no se han considerado en la investigación, enriqueciendo de esta manera la información sobre el tema.

A partir de lo formulado anteriormente se plantea la siguiente pregunta de investigación, ¿Qué influencia ejerce la RE sobre las estrategias de afrontamiento al estrés ante la situación de pandemia en universitarios de Lima?

\section{Objetivos}

El objetivo general de esta investigación fue determinar si existe una influencia significativa de la RE sobre las estrategias de afrontamiento al estrés ante la situación de pandemia en universitarios de Lima.

Con respecto a los objetivos específicos, estos se produjeron en base a la influencia de la RE sobre las estrategias de afrontamiento al estrés, estableciéndose las siguientes:

- Identificar si existe una influencia significativa de la dimensión reevaluación cognitiva y supresión sobre la estrategia de afrontamiento activo en universitarios de Lima.

- Identificar si existe una influencia significativa de la dimensión reevaluación cognitiva y supresión sobre la estrategia de planificación en universitarios de Lima.

- Identificar si existe una influencia significativa de la dimensión reevaluación cognitiva y supresión sobre la estrategia de supresión de actividades en universitarios de Lima.

- Identificar si existe una influencia significativa de la dimensión reevaluación cognitiva y supresión sobre la estrategia de contención del afrontamiento en universitarios de Lima.

- Identificar si existe una influencia significativa de la dimensión reevaluación cognitiva y supresión sobre la estrategia de búsqueda de soporte social por razones instrumentales en universitarios de Lima. 
- Identificar si existe una influencia significativa de la dimensión reevaluación cognitiva y supresión sobre la estrategia de búsqueda de soporte social por razones emocionales en universitarios de Lima.

- Identificar si existe una influencia significativa de la dimensión reevaluación cognitiva y supresión sobre la estrategia de reinterpretación positiva y crecimiento en universitarios de Lima.

- Identificar si existe una influencia significativa de la dimensión reevaluación cognitiva y supresión sobre la estrategia de afrontamiento religioso en universitarios de Lima.

- Identificar si existe una influencia significativa de la dimensión reevaluación cognitiva y supresión sobre la estrategia de aceptación en universitarios de Lima.

- Identificar si existe una influencia significativa de la dimensión reevaluación cognitiva y supresión sobre la estrategia de enfocar y liberar emociones en universitarios de Lima.

- Identificar si existe una influencia significativa de la dimensión reevaluación cognitiva y supresión sobre la estrategia de desentendimiento mental en universitarios de Lima.

- Identificar si existe una influencia significativa de la dimensión reevaluación cognitiva y supresión sobre la estrategia de desentendimiento conductual en universitarios de Lima.

- Identificar si existe una influencia significativa de la dimensión reevaluación cognitiva y supresión sobre la estrategia de negación en universitarios de Lima.

- Identificar si existe una influencia significativa de la dimensión reevaluación cognitiva y supresión sobre la estrategia de uso de sustancias en universitarios de Lima.

- Identificar si existe una influencia significativa de la dimensión reevaluación cognitiva y supresión sobre la estrategia de uso del humor en universitarios de Lima.

\section{Hipótesis de investigación}

Como hipótesis general de trabajo, se espera que la RE influya de forma significativa, positiva y negativamente, sobre las estrategias de afrontamiento al estrés. En lo que se refiere a las hipótesis específicas se plantea lo siguiente:

- La reevaluación cognitiva influye de forma significativa positivamente y la supresión negativamente sobre la estrategia de afrontamiento activo. 
- La reevaluación cognitiva influye de forma significativa positivamente y la supresión negativamente sobre la estrategia de planificación.

- La reevaluación cognitiva influye de forma significativa positivamente y la supresión negativamente sobre la estrategia de supresión de actividades.

- La reevaluación cognitiva influye de forma significativa positivamente y la supresión negativamente sobre la estrategia de contención del afrontamiento.

- La reevaluación cognitiva influye de forma significativa positivamente y la supresión negativamente sobre la estrategia de búsqueda de soporte social por razones instrumentales.

- La reevaluación cognitiva influye de forma significativa, positiva y la supresión negativamente sobre la estrategia de búsqueda de soporte social por razones emocionales.

- La reevaluación cognitiva influye de forma significativa positivamente y la supresión negativamente sobre la estrategia de reinterpretación positiva y crecimiento.

- La reevaluación cognitiva influye de forma significativa positivamente y la supresión negativamente sobre la estrategia de afrontamiento religioso.

- La reevaluación cognitiva influye de forma significativa positivamente y la supresión negativamente sobre la estrategia de aceptación.

- La reevaluación cognitiva influye de forma significativa positivamente y la supresión negativamente sobre la estrategia de enfocar y liberar emociones.

- La reevaluación cognitiva influye de forma significativa negativamente y la supresión positivamente sobre la estrategia de desentendimiento mental.

- La reevaluación cognitiva influye de forma significativa negativamente y la supresión positivamente sobre la estrategia de desentendimiento conductual.

- La reevaluación cognitiva influye de forma significativa negativamente y la supresión positivamente sobre la estrategia de negación.

- La reevaluación cognitiva influye de forma significativa negativamente y la supresión positivamente sobre la estrategia de uso de sustancias.

- La reevaluación cognitiva influye de forma significativa negativamente y la supresión positivamente sobre la estrategia de uso del humor. 


\section{Método}

\section{Tipo y diseño de investigación}

La presente investigación utiliza una estrategia asociativa para determinar la relación funcional entre dos variables; asimismo, el diseño es predictivo ya que se desea determinar la influencia que ejerce la RE (variable independiente o predictor) sobre las estrategias de afrontamiento al estrés (variable dependiente o criterio) ante la situación de pandemia en universitarios de Lima (Ato, López, \& Benavente, 2013).

\section{Participantes}

En lo que respecta al cálculo del tamaño de la muestra, se utilizó el software G*Power 3.1.7 (Faul, Erdfelder, Lang \& Buchner, 2007), la estimación muestral se basó en la magnitud del efecto esperada, error tipo I (alfa) y error tipo II (1- beta). Se consideró un poder estadístico de potencia .95 y una magnitud del efecto esperada de .193 , se determinó que la muestra mínima que se necesita es de 282 estudiantes universitarios.

Asimismo, los criterios de inclusión que se tomaron en cuenta son los siguientes: participar de forma voluntaria en el estudio, encontrarse entre las edades de 18 a 29 años, es decir, ser mayores de edad, estar estudiando en una universidad privada y encontrarse entre el 3er y 8 vo ciclo. Por otro lado, entre los criterios de exclusión se especifica: el ser menor de edad, estar estudiando en una universidad fuera de Lima, ser estudiante de un instituto, encontrarse estudiando una carrera técnica o ser de traslado externo; dichos criterios se consideraron por que la vivencia de estas personas podría ser diferente ya que existe otros factores externos que pueden influir en cómo perciben y actúan en ciertas situaciones; por ejemplo, un estudiante de traslado externo, además de afrontar tareas académicas tiene que afrontar el proceso de adaptación a una institución nueva, por lo cual va estar más estresado que un estudiante regular.

A partir de un muestreo no probabilístico, la muestra estuvo conformada por 333 jóvenes entre hombres (24.9\%) y mujeres (74.8\%), pertenecientes a una universidad privada de Lima; con edades comprendidas entre los 18 y 29 años $(\mathrm{M}=22.2 ; \mathrm{DE}=2.94)$ provenientes de Lima (79.9\%), en mayor porcentaje solteros (97\%) y sin empleo (70.3\%). Asimismo, los participantes en su mayoría se encontraban cursando el 8 vo ciclo (52.9\%) de distintas carreras, predominando la carrera de psicología $(70.3 \%)$.

\section{Instrumentos}


La recolección de datos se realizó a partir de dos instrumentos de evaluación, el primero de ellos es el Cuestionario de Modos de Afrontamiento al Estrés (COPE; Carver, Scheier, \& Weintraub, 1989); para la presente investigación se utilizó la versión adaptada en universitarios de Lima realizado por Cassaretto y Chau (2016), la cual evidenció índices de alfa de Cronbach adecuados en las dimensiones del instrumento, que oscilan entre .53 a .91; de igual forma, en el análisis factorial se obtuvo una varianza del $65.15 \%$ para los 15 factores. El cuestionario emplea una escala de tipo Likert que va del 1 al 4 (Casi nunca lo hago, a veces hago esto, usualmente hago esto y lo hago con mucha frecuencia); cuenta con un total de 60 ítems que miden 15 dimensiones, las cuales están agrupadas en tres estilos de afrontamiento: Centrado en el problema (afrontamiento activo $\left[\alpha=.593 ; \mathrm{r}_{\mathrm{ii}}=.267\right]$ planificación $\left[\alpha=.707 ; \mathrm{r}_{\mathrm{ii}}=.376\right]$, supresión de actividades $\left[\alpha=.514 ; \mathrm{r}_{\mathrm{ii}}=.209\right]$, contención del afrontamiento $\left[\alpha=.555 ; \mathrm{r}_{\mathrm{ii}}=.238\right]$ y búsqueda de soporte social por razones instrumentales $\left[\alpha=.787\right.$; $\left.\mathrm{r}_{\mathrm{ii}}=.480\right]$ ); asimismo, se encuentra el afrontamiento centrado en la emoción (Búsqueda de soporte social por razones emocionales $\left[\alpha=.806 ; \mathrm{r}_{\mathrm{ii}}=.509\right]$, reinterpretación positiva y crecimiento $\left[\alpha=.692 ; \mathrm{r}_{\mathrm{ii}}=.359\right]$, afrontamiento religioso $\left[\alpha=.855 ; \mathrm{r}_{\mathrm{ii}}=.596\right]$, aceptación $\left[\alpha=.581 ; \mathrm{r}_{\mathrm{ii}}=.257\right]$ y enfocar y liberar emociones $\left.\left[\alpha=.650 ; r_{i i}=.317\right]\right)$; por último, se encuentra el centrado en la evitación (desentendimiento mental $\left[\alpha=.350 ; \mathrm{r}_{\mathrm{ii}}=.138\right]$, desentendimiento conductual $\left[\alpha=.626 ; \mathrm{r}_{\mathrm{ii}}=\right.$ .295], negación $\left[\alpha=.681 ; \mathrm{r}_{\mathrm{ii}}=.348\right]$, uso de sustancias $\left[\alpha=.859 ; \mathrm{r}_{\mathrm{ii}}=.604\right]$ y uso del humor $[\alpha$ $\left.\left.=.888 ; \mathrm{r}_{\mathrm{ii}}=.666\right]\right) ;$ los alfa considerados para cada dimensión del instrumento fueron obtenidos con datos propios.

El segundo instrumento es el Cuestionario de Regulación Emocional (ERQ; Gross \& John, 2003); para este estudio se utilizó la adaptación (ERQP) realizada por Gargurevich y Matos (2010), en el cual se obtuvo un alfa de Cronbach de .72 para la dimensión de reevaluación cognitiva mientras que para la dimensión de supresión se consiguió un alfa de Cronbach de .74; respecto al análisis factorial confirmatorio se demostró la estructura bifactorial del instrumento, asimismo se comprobó la validez convergente y divergente. El cuestionario está conformado por 10 ítems agrupados en dos dimensiones: la supresión $\left(\alpha=.787 ; \mathrm{r}_{\mathrm{ii}}=.480\right)$ dividido en 4 ítems y la reevaluación cognitiva $\left(\alpha=.80 ; \mathrm{r}_{\mathrm{ii}}=.40\right)$ compuesta por 6 ítems; asimismo, utiliza una escala de tipo Likert que va del 1 (Totalmente de acuerdo) al 7 (Totalmente en desacuerdo); los alfa considerados para ambas dimensiones del instrumento fueron obtenidos con datos propios. 


\section{Procedimientos}

Previo a la recolección de datos, se pidió permiso a los autores de las adaptaciones de los instrumentos para poder hacer uso de estos, solo se obtuvo respuesta de uno de ellos, a pesar de ello, al no ser una escala con derechos comerciales se decidió utilizar el cuestionario que se encontraba dentro del artículo de la adaptación. Una vez obtenidos los instrumentos, se solicitó el permiso a la universidad para poder evaluar a los estudiantes; teniendo la aprobación, se les envió un formulario a los estudiantes con las encuestas a sus correos institucionales para que puedan realizarlo. En vista de la baja tasa de respuesta, se procedió a enviar el formulario por otros medios como redes sociales, para poder llegar a más personas y obtener la cantidad deseada, ya no se pidió permiso a otras instituciones, pero si se utilizó el consentimiento informado.

En el formulario se encuentra inicialmente un consentimiento informado (apéndice 1) en el cual se especifica que toda la información es confidencial y solo para fines académicos, de igual forma, se les indica que la encuesta es voluntaria y anónima (American Psychological Association, 2010), asimismo, se menciona el objetivo de la investigación, el tiempo que tardará (10 minutos) y un correo mediante el cual se pueden contactar en caso tenga alguna consulta. Debido a que es una evaluación anónima, no se realizó una devolución de la información, pero se les indico que, si deseaban conocer los resultados de la investigación, se les enviaría una vez terminada. Seguido del consentimiento informado, se encuentra una ficha sociodemográfica (apéndice 2) y los dos instrumentos.

Finalmente, una vez obtenidos las encuestas de los participantes, se procedió a pasar todas las respuestas a una base de datos en Excel para posteriormente llevarlas al programa SPSS en el cual se continuó con el respectivo análisis.

\section{Análisis de datos}

Para el análisis de datos se utilizó el programa estadístico Jamovi versión 1.2 (The jamovi Project, 2020); primeramente, se procedió a realizar un análisis de confiabilidad a partir del coeficiente alfa y la correlación inter-ítem promedio $\left(\mathrm{r}_{i i}\right)$ para la confiabilidad de las puntuaciones; debido a que las dimensiones evalúan constructos amplios, $\alpha$ con valores alrededor de $.60 \mathrm{y} \mathrm{r}_{\mathrm{ii}}$ en el rango de .15 y .20 son aceptables (Clark \& Watson, 1995). Asimismo, 
mediante la base de datos se realizó un análisis estadístico descriptivo por cada una de las variables de estudio.

Los resultados se interpretaron a partir de la magnitud del efecto (ME), siendo $\mathrm{r} \geq .20$ considerado como significativo; de igual forma, previo a realizar el análisis de regresión lineal se tomaron en cuenta algunos supuestos estadísticos; primeramente, la normalidad, para analizar si la distribución de las variable se encuentra dentro de un rango razonable mediante la curtosis (+/- 3); la linealidad se valoró explorando la asociación entre las variables a partir de la correlación de Pearson entre las dimensiones de RE y estrategias de afrontamiento al estrés; la independencia de errores, se analizó con la magnitud del estadístico de Durbin-Watson, considerando como aceptable los valores entre 1.5 y 2.5 ; por último, la multicolinealidad se obtuvo con los indicadores de tolerancia y el factor de inflación de varianza (FIV), considerando con ausencia de multicolinealidad a los valores mayores que .10 y menos que 10 respectivamente (Vilà Baños, Torrado-Fonseca, \& Reguant-Álvarez, 2019).

Seguidamente se procedió a realizar un análisis de regresión lineal para el contraste de las hipótesis específicas, es decir, la influencia de la variable RE sobre las estrategias de afrontamiento al estrés. Se consideró un $R^{2}$ insignificante (valores menores de .04), bajo (entre .04 y .25), moderado (entre .25 y .64) y fuerte (mayores que .64), de igual forma, para la beta estandarizada se consideró un $\beta$ insignificante (valores menores a .20), bajo (entre .20 y .50), moderado (entre .50 y .80) y fuerte (mayores que .80) (Ferguson, 2009). Por último, la hipótesis general se evaluó a partir de los hallazgos de las hipótesis específicas dado que no es posible hacerlo de manera directa por la naturaleza multidimensional de los constructos estudiados. 


\section{Resultados}

\section{Análisis preliminar descriptivo}

Respecto al análisis descriptivo, el promedio de la media respecto a la reevaluación cognitiva $(M=29.4)$ supera la media teórica mientras que para la supresión $(M=14.9)$ no; por otro lado, en lo que respecta a las estrategias, la mayor parte de ellas obtuvieron valores que superan la media teórica; sin embargo, algunos como el afrontamiento religioso $(\mathrm{M}=6.86)$, el desentendimiento conductual $(\mathrm{M}=6.75)$, la negación $(\mathrm{M}=6.86)$ y el uso de sustancias $(\mathrm{M}=$ 5.06) estuvieron por debajo del promedio teórico (Tabla 1).

En lo que se refiere al coeficiente de confiablidad se puede observar que algunos valores de las dimensiones del COPE están por debajo del valor aceptable (Tabla 1); debido a ello, se procedió a realizar una correlación inter-ítem promedio $\left(\mathrm{r}_{\mathrm{ii}}\right)$; las dimensiones en su gran mayoría obtuvieron un coeficiente aceptable; a pesar de ello, se tuvo que eliminar la dimensión de desentendimiento mental $\left(\alpha=.350 ; r_{i i}=.138\right)$ debido a su baja confiabilidad.

\section{Tabla 1}

Análisis descriptivo de las dimensiones de RE y estrategias de afrontamiento

\begin{tabular}{lccccc}
\hline & $M$ & $D E$ & $g_{1}$ & $g_{2}$ & $\alpha$ \\
\hline Regulación emocional & & & & & \\
Reevaluación cognitiva & 29.4 & 6.36 & -.508 & .693 & .800 \\
Supresión & 14.9 & 5.29 & -.022 & -.587 & .787 \\
Estrategias de afrontamiento & & & & & \\
Estilo centrado en el problema & & & & & \\
Afrontamiento activo & 10.0 & 2.04 & .085 & -.136 & .593 \\
Planificación & 10.7 & 2.22 & .146 & -.250 & .707 \\
Supresión de actividades & 9.20 & 2.09 & .230 & .110 & .514 \\
Contención del afrontamiento & 9.73 & 2.09 & .213 & .168 & .555 \\
Búsqueda de SS razones instrumentales & 9.70 & 2.52 & .104 & -.470 & .787
\end{tabular}




\begin{tabular}{llllll} 
Estilo centrado en la emoción & & & & \\
Búsqueda de SS razones emocionales & 9.56 & 2.95 & .163 & -.514 & .806 \\
Re-interpretación positiva y crecimiento & 11.2 & 2.24 & -.019 & -.076 & .692 \\
Afrontamiento religioso & 6.86 & 2.30 & .731 & .387 & .855 \\
Aceptación & 10.4 & 2.18 & .499 & -.077 & .581 \\
Enfocar y liberar emociones & 8.52 & 2.38 & .288 & -.170 & .650 \\
& & & & & \\
Estilo evitativo & & & & .350 \\
Desentendimiento mental & 9.42 & 2.14 & .329 & .131 & .626 \\
Desentendimiento conductual & 6.75 & 2.09 & .669 & -.081 & .681 \\
Negación & 6.86 & 2.30 & .731 & .387 \\
Uso del humor & 8.70 & 3.12 & .302 & -.453 & .888 \\
Uso de sustancias & 5.06 & 2.09 & 2.27 & 5.22 & .859 \\
\hline
\end{tabular}

Nota: $\mathrm{n}=333 ; M=$ Media; $D E=$ Desviación estándar; $g_{1}=$ Asimetría; $g_{2}=$ Curtosis

\section{Contraste de hipótesis}

En lo que se refiere a las hipótesis enfocadas en las estrategias de afrontamiento asociadas al estilo centrado en el problema, para evaluar la influencia de la RE sobre las estrategias de afrontamiento activo, planificación, supresión de actividades, contención del afrontamiento y búsqueda de soporte social por razones instrumentales; primero se tomaron en cuenta los supuestos estadísticos mediante los cuales se obtuvo evidencia favorable en cuanto a la normalidad, en la medida que la distribución de las variables se encuentra dentro de un rango aceptable (Tabla 1); la asociación lineal entre variables se verificó porque en el estilo centrado en el problema, la dimensión de reevaluación cognitiva evidencia una correlación positiva y significativa entre las dimensiones de afrontamiento activo, planificación, supresión de actividades y contención del afrontamiento; mientras que para la dimensión de supresión no se obtuvo correlaciones estadísticamente significativas (Tabla 2), en cuanto a la independencia de los errores, el estadístico de Durbin-Watson fue aceptable con cada una de las estrategias de 
afrontamiento activo (1.85), planificación (1.99), supresión de actividades (1.83), contención de afrontamiento (1.80) y búsqueda de SS razones instrumentales (1.25); por último, hubo ausencia de multicolinealidad en vista de los valores para reevaluación cognitiva (tolerancia $=.997$; FIV $=1.00)$ y supresión $($ tolerancia $=.997 ;$ FIV $=1.00)$.

\section{Tabla 2}

Análisis correlacional entre las dimensiones de RE y las estrategias del estilo centrado en el problema

\begin{tabular}{lccc}
\hline & & Reevaluación cognitiva & Supresión \\
\hline Estilo centrado en el problema & & & \\
Afrontamiento activo & $r$ & .281 & -.153 \\
& $p$ & .001 & .005 \\
Planificación & $r$ & .319 & -.142 \\
& $p$ & .001 & .009 \\
Supresión de actividades & & & .073 \\
& $r$ & .218 & .186 \\
Contención del afrontamiento & $p$ & .001 & .016 \\
& & & .764 \\
Búsqueda de SS razones instrumentales & $r$ & .280 & -.191 \\
& $p$ & .001 & .001 \\
\hline
\end{tabular}

Nota: $\mathrm{n}=333 ; r>.20$

En cuanto al análisis principal, se encontró una influencia conjunta estadísticamente significativa y de magnitud baja de la reevaluación cognitiva y supresión sobre el afrontamiento activo $\left(\mathrm{F}[2,330]=19.9 ; \mathrm{p}<.001 ; \mathrm{R}^{2}=.102\right)$, la planificación $\left(\mathrm{F}[2,330]=24.1 ; \mathrm{p}<.001 ; \mathrm{R}^{2}=\right.$ $.122)$, supresión de actividades $\left(\mathrm{F}[2,330]=8.87 ; \mathrm{p}<.001 ; \mathrm{R}^{2}=.045\right)$, contención de afrontamiento $\left(\mathrm{F}[2,330]=14.1 ; \mathrm{p}<.001 ; \mathrm{R}^{2}=.072\right)$ y búsqueda de $\mathrm{SS}$ por razones instrumentales $\left(\mathrm{F}[2,330]=12.4 ; \mathrm{p}<.001 ; \mathrm{R}^{2}=.064\right)$. Específicamente, solo la reevaluación cognitiva influye de forma significativa y baja sobre el afrontamiento activo, planificación, supresión de actividades y contención de afrontamiento $(\beta>|.20|)$, mientras que la supresión sobre la estrategia de búsqueda de SS por razones instrumentales $(\beta>|.20|)$ (Tabla 3). En ese sentido, en vista de la evidencia presentada, las hipótesis de investigación enfocadas 
específicamente en las estrategias de afrontamiento activo, planificación, supresión de actividades, contención del afrontamiento y búsqueda de soporte social por razones instrumentales, reciben respaldo parcial.

\section{Tabla 3}

Análisis de la influencia de las dimensiones de la RE sobre las estrategias del estilo centrado en el problema

\begin{tabular}{|c|c|c|c|c|c|c|c|c|c|c|}
\hline & \multicolumn{2}{|c|}{$\begin{array}{c}\text { Afrontamiento } \\
\text { activo }\end{array}$} & \multicolumn{2}{|c|}{ Planificación } & \multicolumn{2}{|c|}{$\begin{array}{l}\text { Supresión de } \\
\text { actividades }\end{array}$} & \multicolumn{2}{|c|}{$\begin{array}{l}\text { Contención de } \\
\text { afrontamiento }\end{array}$} & \multicolumn{2}{|c|}{$\begin{array}{l}\text { Búsqueda de } \\
\text { SS razones } \\
\text { instrumentales }\end{array}$} \\
\hline & $\beta$ & $t$ & $\beta$ & $t$ & $\beta$ & $t$ & $\beta$ & $t$ & $\beta$ & $t$ \\
\hline $\begin{array}{l}\text { Reevaluación } \\
\text { cognitiva }\end{array}$ & .290 & $5.58^{* \star}$ & .328 & $6.37^{\star \star}$ & .214 & $3.99^{\star *}$ & .280 & $5.29^{\star \star}$ & .183 & $3.43^{\star \star}$ \\
\hline Supresión & -.169 & $-3.25^{\star *}$ & -.161 & $-3.12^{*}$ & .060 & 1.13 & .001 & .013 & -.202 & $3.79^{* \star}$ \\
\hline
\end{tabular}

Nota: $* p<.05 ; * * p<.001$

\section{Contraste de hipótesis}

Respecto a las hipótesis enfocadas en las estrategias de afrontamiento asociadas al estilo centrado en la emoción, previo a evaluar la influencia de la RE sobre las estrategias de búsqueda de soporte social por razones emocionales, reinterpretación positiva y crecimiento, afrontamiento religioso, aceptación y enfocar y liberar emociones; se consideró los supuestos estadísticos a partir de los cuales se obtuvo evidencia favorable en cuanto a la normalidad, en la medida que la distribución de las variables se encuentra dentro de un rango aceptable (Tabla 1); la asociación lineal entre variables se verificó porque la reevaluación cognitiva obtuvo un correlación positiva y significativa con las dimensiones de aceptación y reinterpretación positiva y crecimiento; asimismo, la supresión presentó una correlación positiva y significativa con el afrontamiento religioso; mientras que con la dimensión de búsqueda de soporte social por razones emocionales y reinterpretación positiva y crecimiento se muestra una correlación negativa y significativa (Tabla 4), en cuanto a la independencia de los errores, el estadístico de Durbin-Watson es aceptable con cada una de las estrategias de búsqueda de SS razones emocionales (1.90), reinterpretación positiva y crecimiento (2.00), afrontamiento religioso (1.95), aceptación (1.92) y enfocar y liberar emociones (1.77); asimismo, hubo ausencia de 
multicolinealidad debido a que los valores para reevaluación cognitiva (tolerancia = .997; FIV $=1.00$ ) y supresión (tolerancia $=.997 ; \mathrm{FIV}=1.00$ ) fueron aceptables.

\section{Tabla 4}

Análisis correlacional entre las dimensiones de RE y las estrategias del estilo centrado en la emoción

\begin{tabular}{llll}
\hline & & Reevaluación cognitiva & Supresión \\
\hline Estilo centrado en la emoción & & & \\
Búsqueda de SS razones emocionales & $r$ & .084 & .334 \\
& $p$ & .128 & .001 \\
Reinterpretación positiva y crecimiento & $r$ & & .259 \\
& $p$ & .343 & .001 \\
Afrontamiento religioso & & .001 & .270 \\
& $r$ & .032 & .001 \\
Aceptación & $p$ & .563 & -.059 \\
& & & .279 \\
Enfocar y liberar emociones & $r$ & .240 & -.020 \\
& $p$ & .001 & .716 \\
\hline
\end{tabular}

Nota: $\mathrm{n}=333 ; r>.20$

A partir del análisis de regresión, se evidencia una influencia conjunta estadísticamente significativa y de magnitud baja de la reevaluación cognitiva y supresión sobre la búsqueda de SS por razones emocionales $\left(\mathrm{F}[2,330]=23.0 ; \mathrm{p}<.001 ; \mathrm{R}^{2}=.117\right)$, reinterpretación positiva $\mathrm{y}$ crecimiento $\left(\mathrm{F}[2,330]=40.2 ; \mathrm{p}<.001 ; \mathrm{R}^{2}=.191\right)$, afrontamiento religioso $(\mathrm{F}[2,330]=13.1$; $\left.\mathrm{p}<.001 ; \mathrm{R}^{2}=.067\right)$ y aceptación $\left(\mathrm{F}[2,330]=8.87 ; \mathrm{p}<.001 ; \mathrm{R}^{2}=.057\right)$. Con respecto al análisis sobre cada dimensión, solo la reevaluación cognitiva influye de forma significativa y baja sobre la reinterpretación positiva y crecimiento, así como la aceptación $(\beta>|.20|)$; mientras que la supresión sobre la búsqueda de SS por razones emocionales, reinterpretación positiva y crecimiento, y afrontamiento religioso $(\beta>|.20|)$ Finalmente, no se encontró una influencia estadísticamente significativa $(\mathrm{F}[2,330]=0.186 ; \mathrm{p}<.001)$ de la reevaluación cognitiva $\mathrm{y}$ supresión sobre la dimensión de enfocar y liberar emociones debido a que se obtuvo una magnitud insignificante $\left(\mathrm{R}^{2}=.004\right)$ (Tabla 5). En ese sentido, en vista de la evidencia 
presentada, solo las hipótesis de investigación enfocadas en las estrategias de búsqueda de soporte social por razones emocionales, reinterpretación positiva y crecimiento, así como la aceptación reciben respaldo parcial, mientras que las hipótesis referidas a las estrategias de afrontamiento religioso y enfocar y liberar emociones no reciben respaldo.

\section{Tabla 5}

Análisis de la influencia de las dimensiones de la RE sobre las estrategias del estilo centrado en la emoción

\begin{tabular}{|c|c|c|c|c|c|c|c|c|c|c|}
\hline & \multicolumn{2}{|c|}{$\begin{array}{c}\text { Búsqueda de SS } \\
\text { razones } \\
\text { emocionales }\end{array}$} & \multicolumn{2}{|c|}{$\begin{array}{c}\text { Reinterpretación } \\
\text { positiva y } \\
\text { crecimiento }\end{array}$} & \multicolumn{2}{|c|}{$\begin{array}{c}\text { Afrontamiento } \\
\text { religioso }\end{array}$} & \multicolumn{2}{|c|}{ Aceptación } & \multicolumn{2}{|c|}{$\begin{array}{l}\text { Enfocar y } \\
\text { liberar } \\
\text { emociones }\end{array}$} \\
\hline & $\beta$ & $t$ & $\beta$ & $t$ & $\beta$ & $t$ & $\beta$ & $t$ & $\beta$ & $t$ \\
\hline $\begin{array}{l}\text { Reevaluación } \\
\text { cognitiva }\end{array}$ & .103 & $1.99^{\star}$ & .359 & $7.26^{\star *}$ & .016 & .314 & .244 & $4.58^{* *}$ & .026 & -.488 \\
\hline Supresión & -.340 & $-6.58^{* *}$ & -.280 & $-5.66^{* *}$ & .269 & $5.07^{\star *}$ & .073 & -1.37 & .018 & -.336 \\
\hline
\end{tabular}

Nota: $* p<.05 ; * * p<.001$

\section{Contraste de hipótesis}

En lo que se refiere a las hipótesis enfocadas en las estrategias asociadas al estilo evitativo, previo a evaluar la influencia de la RE sobre las estrategias de desentendimiento conductual, negación, uso de sustancias y uso del humor; se tomaron en cuenta los supuestos estadísticos mediante los cuales se obtuvo evidencia favorable en cuanto a la normalidad, en la medida que la distribución de las variables se encuentra dentro de un rango aceptable, excepto la dimensión de uso de sustancias (Tabla 1); la asociación lineal entre variables se verificó porque la reevaluación cognitiva muestra una correlación positiva y significativa con la dimensión de uso del humor; mientras que la supresión se correlaciona positiva y significativamente con las dimensiones de desentendimiento conductual y negación (Tabla 6), en cuanto a la independencia de los errores, el estadístico de Durbin-Watson fue aceptable con cada una de las estrategias de desentendimiento conductual (1.91), negación (1.95), uso del humor (1.90) y uso de sustancias (1.97); asimismo, hubo ausencia de multicolinealidad debido a que los valores para reevaluación cognitiva (tolerancia $=.997$; FIV $=1.00$ ) y supresión (tolerancia $=.997 ; \mathrm{FIV}=1.00)$ fueron aceptables.

\section{Tabla 6}


Análisis correlacional entre las dimensiones de RE y las estrategias del estilo evitativo

\begin{tabular}{llll}
\hline & & Reevaluación cognitiva & Supresión \\
\hline Estilo evitativo & & & .312 \\
Desentendimiento conductual & $r$ & -.110 & .001 \\
& $p$ & .044 & .270 \\
Negación & & & .001 \\
& $r$ & .032 & .089 \\
Uso del humor & $p$ & .563 & .103 \\
& & & .201 \\
Uso de sustancias & $r$ & .001 & .055 \\
& $p$ & & .313 \\
\hline
\end{tabular}

Nota: $\mathrm{n}=333 ; r>.20$

Mediante el análisis de regresión lineal, se halló una influencia conjunta estadísticamente significativa y de magnitud baja de la reevaluación cognitiva y supresión sobre desentendimiento conductual $\left(\mathrm{F}[2,330]=21.2 ; \mathrm{p}<.001 ; \mathrm{R}^{2}=.109\right)$, negación $(\mathrm{F}[2,330]=$ $\left.13.1 ; \mathrm{p}<.001 ; \mathrm{R}^{2}=.067\right)$, y uso del humor $\left(\mathrm{F}[2,330]=8.05 ; \mathrm{p}<.001 ; \mathrm{R}^{2}=.040\right)$. Concretamente, solo la reevaluación cognitiva influye de forma significativa y baja sobre el uso del humor $(\beta>|.20|)$; mientras que la supresión sobre el desentendimiento conductual y la negación $(\beta>|.20|)$. Por otra parte, no se encontró una influencia significativa $(\mathrm{F}[2,330]=$ 0.513; $\mathrm{p}$ <.599) de la reevaluación cognitiva y supresión sobre la dimensión de uso de sustancias ya que se obtuvo una magnitud insignificante $\left(\mathrm{R}^{2}=.002\right)$ (Tabla 7). En ese sentido, en vista de la evidencia presentada, las hipótesis de investigación enfocadas en el desentendimiento conductual y negación reciben respaldo parcial; mientras que las hipótesis referidas al uso del humor y uso de sustancias no reciben respaldo.

\section{Tabla 7}

Análisis de la influencia de las dimensiones de la RE sobre las estrategias del estilo evitativo

\begin{tabular}{ccccccc}
\hline $\begin{array}{c}\text { Desentendimiento } \\
\text { conductual }\end{array}$ & Negación & Uso del humor & $\begin{array}{c}\text { Uso de } \\
\text { sustancias }\end{array}$ \\
\hline$\beta$ & $t$ & $\beta$ & $t$ & $\beta$ & $t$ & $\beta$
\end{tabular}




\begin{tabular}{lcccccccc}
\hline $\begin{array}{l}\text { Reevaluación } \\
\text { cognitiva }\end{array}$ & -.128 & $-2.47^{*}$ & .016 & .314 & .196 & $3.65^{* *}$ & .004 & .085 \\
Supresión & .319 & $6.16^{* *}$ & .269 & $5.07^{* *}$ & .078 & 1.46 & .055 & 1.00 \\
& & & & & & & & \\
\hline Nota: $* p<.05 ; * * p<.001$ & & & & & &
\end{tabular}

\section{Sobre la hipótesis general}

Finalmente, en lo que respecta a la hipótesis general la cual fue contrastada a partir de los hallazgos de las hipótesis específicas, indica que la RE influye de forma significativa, positiva y negativamente, sobre las estrategias de afrontamiento al estrés. En ese sentido, recibe respaldo parcial ya que a pesar de que no se aceptó todas las hipótesis específicas, las dimensiones de la RE sí influyen en alguna de las estrategias de afrontamiento al estrés. 


\section{Discusión}

La situación de la pandemia debido al COVID-19 ha ocasionado distintas consecuencias en la salud mental de los jóvenes, como puede ser la presencia de emociones más intensas y de mayor duración, lo cual ha influido tanto en el ámbito académico como en otras situaciones de la vida cotidiana. A partir de ello, la forma de afrontar estas situaciones se puede ver influida según cómo los individuos regulan sus emociones. Mediante lo indicado anteriormente, se planteó como objetivo determinar si existe una influencia significativa de la RE sobre las estrategias de afrontamiento al estrés ante la situación de pandemia en universitarios de Lima.

En cuanto a la hipótesis especifica 1, la cual indica que la reevaluación cognitiva influye de forma significativa positivamente y la supresión negativamente sobre la estrategia de afrontamiento activo; se pudo encontrar que solo la revaluación cognitiva influye positivamente en el afrontamiento activo, por lo que existe respaldo parcial; dado que la reevaluación cognitiva permite tener una experiencia menos aversiva frente a algunos estímulos debido a que se modifica el proceso sobre la cual se da una emoción, los jóvenes podrán afrontar una situación como, por ejemplo, las evaluaciones en modalidad virtual, para evitar o disminuir consecuencias negativas como puede ser desaprobar (afrontamiento activo). Por otro lado, la hipótesis especifica 2 y 3 , refieren que la reevaluación cognitiva influye de forma significativa positivamente y la supresión negativamente sobre las estrategias de planificación y supresión de actividades; a partir de ello, se encontró que solo la reevaluación cognitiva influye positivamente sobre la planificación y la supresión de actividades, por lo que se obtuvo un respaldo parcial; al no involucrar muchos recursos cognitivos o fisiológicos para regular sus emociones (reevaluación cognitiva), esto se verá influido cuando la persona planifique pasos a seguir, acciones o maneras de afrontar una situación estresante (planificación), así como, al momento de tener los recursos necesarios para concentrarse y hacer los esfuerzos necesarios para afrontar un evento en el ámbito académico o personal que produzca estrés (supresión de actividades). Asimismo, la hipótesis especifica 4, indica que la reevaluación cognitiva influye de forma significativa positivamente y la supresión negativamente sobre la estrategia de contención del afrontamiento, a partir de ello se encontró que la reevaluación cognitiva influye de manera positiva en la contención de afrontamiento por lo que la hipótesis planteada recibe 
un respaldo parcial; al gestionar mejor sus emociones los jóvenes actuarán de manera más precisa y apropiada ante un evento estresor (contención de afrontamiento).

Por último, respecto a la hipótesis especifica 5, la cual refiere que la reevaluación cognitiva influye de forma significativa positivamente y la supresión negativamente sobre la estrategia de búsqueda de soporte social por razones instrumentales, se obtuvo que la supresión influye negativamente sobre la búsqueda de soporte social por razones instrumentales, debido a ello se encontró solo un respaldo parcial; esto indicaría que al inhibir la expresión de la respuesta emocional produciría una menor comunicación del estado del individuo con su entorno inmediato, lo cual podría influir cuando los jóvenes decidan o no buscar consejos o información para lidiar con un problema, ya sea en amigos, pareja o familiares (búsqueda de soporte social por razones instrumentales).

Respecto a la hipótesis especifica 6, la cual menciona que la reevaluación cognitiva influye de forma significativa positivamente y la supresión negativamente sobre la estrategia de búsqueda de soporte social por razones emocionales, se pudo encontrar que solo la supresión influye negativamente sobre la búsqueda de soporte social por razones emocionales por lo que se obtuvo recibe respaldo parcial; la supresión indica que las personas son más propensas a tener una menor comunicación con su entorno sobre sus sentimientos por lo que presentan una resistencia a buscar ayuda, lo cual no permitiría que los jóvenes busquen un soporte para reducir el impacto emocional producto de una situación estresante, por ejemplo, si la persona no expresa su frustración o angustia por no tener los resultados esperados en un examen, esto hará que se le dificulte más afrontar dicha situación (búsqueda de soporte social por razones emocionales).

Por otro lado, la hipótesis especifica 7 , la cual menciona que la reevaluación cognitiva influye de forma significativa positivamente y la supresión negativamente sobre la estrategia de la reinterpretación positiva y crecimiento, se encontró que si recibe respaldo debido a que la reevaluación cognitiva influye de manera positiva en la reinterpretación positiva y crecimiento, mientras que la supresión de manera negativa; esto se puede explicar en la medida que aquellos jóvenes que usan la reevaluación cognitiva suelen compartir y expresan sus emociones con otras personas ya sea amigos, familiares o pareja, lo que permite una mayor posibilidad de experimentar emociones más positivas disminuyendo el impacto de las negativas. Por ende, la persona evaluará e interpretará un evento, por ejemplo, una nota baja o dificultades en algún 
curso, como una experiencia de aprendizaje (reinterpretación positiva y crecimiento); por otro lado, en la supresión al inhibir la respuesta emocional producto de una situación, a la persona se le dificultará evaluar e interpretar como aprendizaje a dicho evento estresor (reinterpretación positiva y crecimiento).

Asimismo, la hipótesis especifica 8 , indica que la reevaluación cognitiva influye de forma significativa positivamente y la supresión negativamente sobre la estrategia de afrontamiento religioso; a partir de los resultados, se encontró una influencia positiva de la supresión sobre el afrontamiento religioso, lo cual no respalda la hipótesis planteada; a pesar de ello, esto ocurriría porque al inhibir las emociones y no buscar apoyo en otros, la persona se apoyaría en sus creencias religiosas para poder afrontar una situación que excede sus recursos. Por otro lado, la hipótesis especifica 9 menciona que la reevaluación cognitiva influye de forma significativa positivamente y la supresión negativamente sobre la estrategia de aceptación, los resultados indican que existe un respaldo parcial dado que la reevaluación cognitiva influye en la estrategia de aceptación; al tener una mejor regulación emocional va a permitir que la persona sea más consciente y acepte convivir con el evento estresor para cambiar dicha situación de tal forma que, si por ejemplo, un joven llega a desaprobar un curso, la persona aceptará dicha situación pero tratará de cambiar sus acciones la próxima vez, con la finalidad de que no vuelva a experimentar dicha experiencia (aceptación).

Respecto a la hipótesis especifica 11 y 12 se planteó que la reevaluación cognitiva influye de forma significativa negativamente y la supresión positivamente sobre la estrategia de desentendimiento mental y negación; los resultados mencionan que solo existe una influencia positiva de la supresión sobre el desentendimiento conductual y la negación. Al ser menos conscientes y no expresar sus emociones con otras personas, los jóvenes podrían dejar de lado los objetivos que se han visto afectados por la situación estresante, ya que no tienen un respaldo o apoyo que les permita afrontar dicho evento; por ejemplo, una problema o discusión con algún familiar podría influir en que un joven no pueda concentrarse en una actividad que tenía planeado realizar ya que no expresa lo que siente con otra persona (desentendimiento conductual). De igual forma, no expresar la respuesta emocional también podría influir que, ante una situación que exceda los recursos de la persona, esta prefiera negar que dicha situación 
produce estrés por lo que actuará como si no existiera; por ejemplo, las evaluaciones o exámenes, serían postergados y no se estudiaría para evitar la experiencia aversiva (negación).

Por otro lado, se encontró que la reevaluación cognitiva influye de forma positiva en la estrategia de uso del humor, lo cual no respalda la hipótesis planteada; esto se puede deber a que la dimensión de uso del humor se ha visto determinada por factores socioculturales (Cassaretto \& Chau, 2016), debido a ello, dicha dimensión también ha sido ubicada dentro del estilo centrado en la emoción, sin embargo, para fines de agrupar la estrategia, se optó por seguir el modelo de Carver et al. (1989) e incluir la dimensión dentro del estilo evitativo. Finalmente, en lo que se refiere a la estrategia de uso de sustancias, la hipótesis planteada tampoco fue respaldada, a partir de ello, se infiere que, al tener una muestra conformada por jóvenes universitarios, el consumo de sustancias no necesariamente va a ser significativo y, por ende, esto habría influido en los resultados.

Los resultados encontrados coinciden con los hallazgos de otros estudios (Ong \& Thompson, 2019; Piri et al., 2019; Midkiff, Lindsey \& Meadows, 2018; Smrtnik \& Prosen, 2016; Monteiro, Balogun \& Oratile, 2014; Wong et al., 2013; Cabral et al., 2012) que muestran que existe una relación entre la RE y las estrategias de afrontamiento al estrés. Por ejemplo, Smrtnik y Prosen (2016) reportaron que tanto la asociación de la dimensión de reevaluación cognitiva y planificación, así como la supresión con el desentendimiento conductual y la contención de afrontamiento son estadísticamente significativas, aunque en una magnitud baja. De igual forma, Ong y Thompson (2019) encontraron que la reevaluación cognitiva está relacionada de forma significativa y baja con las dimensiones de reinterpretación positiva y crecimiento, afrontamiento activo y planificación, al igual que la supresión con las dimensiones de desentendimiento conductual y reinterpretación positiva y crecimiento. Respecto a la última dimensión mencionada, su significancia fue de magnitud moderada. Dicha diferencia con los resultados obtenidos se podría deber a distintos factores como la cultura, el estudio mencionado se realizó en estudiantes universitarios de China; en países orientales como este se tiene una perspectiva diferente ya que presentan una cultura colectivista, mientras que en países como el Perú los estudios se basan en las características de la sociedad, es decir, desde un enfoque más individualista. 
Asimismo, en otro estudio, con una muestra de universitarios, la reevaluación cognitiva estuvo asociada con las dimensiones de afrontamiento activo, la búsqueda de soporte social por razones emocionales y la reinterpretación positiva y crecimiento, mientras que la supresión solo se relacionó de forma significativa con la búsqueda de soporte social por razones emocionales (Cabral et al., 2012); estos resultados son parecidos a los encontrados, a pesar de ello, difiere en que, en el presente estudio la relación entre la dimensión de reevaluación cognitiva y la búsqueda de soporte social por razones emocionales fue insignificante, esta diferencia podría darse porque la muestra estuvo conformada jóvenes del primer año de la universidad (Cabral et al., 2012), lo cual pudo influir ya que la adaptación al contexto académico es reciente, y los participantes buscarían un mayor apoyo de su entorno cercano.

Por último, las investigaciones indicadas anteriormente no encontraron relación significativa entre las dimensiones de la RE y ciertas estrategias de afrontamiento al estrés (supresión de actividades, búsqueda de soporte social por razones instrumentales, afrontamiento religioso, enfocar y liberar emociones, negación, uso del humor y uso de sustancias) en comparación con algunos de los resultados del presente estudio, lo cual se puede deber a que este estudio se realizó en un contexto diferente debido a la pandemia por la COVID19. A su vez, los distintos estudios fueron realizados en otros países, siendo en su gran mayoría del continente europeo y asiático, lo cual podría influir en cierta medida los resultados obtenidos ya que la persona no percibe o asimila una situación de la misma manera. Por otro lado, algunas investigaciones presentadas en los antecedentes (Ong \& Thompson, 2019; Piri et al., 2019; Midkiff, Lindsey \& Meadows, 2018; Monteiro, Balogun \& Oratile, 2014; Cabral et al., 2012) fueron realizados en estudiantes universitarios lo que pudo haber influido específicamente en los resultados de la dimensión de uso de sustancias ya que el consumo no es significativamente elevado.

Las implicancias prácticas de los hallazgos para el presente estudio podrían verse abordadas a partir de algún programa de intervención aplicado en las universidades, enfocados principalmente en la RE, ya que a pesar de que existe una influencia parcial de dicha variable sobre las estrategias de afrontamiento al estrés, sigue habiendo una influencia en algunas de las estrategias, a partir de ello, podría valorarse la propuesta de Pérez (2014), cuyo programa estuvo dirigido a adultos y se consideró algunos puntos que podrían ser adaptados en el contexto 
académico. Inicialmente, siguiendo los modelos de RE (Gross, 1999), sería importante enseñar a los jóvenes a ser más conscientes y reflexionar sobre su estado emocional; de igual forma, explicar las consecuencias de las emociones en el aspecto fisiológico, cognitivo y conductual, así como brindar un espacio en el que se normalice la expresión de distintas emociones derivadas de conductas y situaciones de la vida cotidiana. También, se les podría enseñar a las jóvenes técnicas de relajación que los ayude durante algún evento a regular sus emociones. Finalmente, una vez culminado el programa, también sería pertinente brindar un taller para que los jóvenes adquieran herramientas para hacer un mejor uso de las estrategias de afrontamiento al estrés.

En cuanto a las fortalezas de este estudio, una de ellas es que se realizó en un contexto diferente, por la situación de pandemia, además de que no existen investigaciones que abarquen ambas variables en Latinoamérica, por lo que el aporte de los resultados brinda información empírica reciente sobre la influencia de la regulación emocional sobre las estrategias de afrontamiento al estrés en jóvenes universitarios.

Sin embargo, la presente investigación cuenta con algunas limitaciones. Como se indicó anteriormente, la recolección de datos no se puedo hacer de forma presencial, por lo que se envió el formulario al correo institucional de los alumnos, al obtener una tasa baja de respuestas se procedió difundirlo por redes sociales lo cual pudo dificultar la selección de jóvenes que cumplieran con los criterios de inclusión establecidos, es por ello que no existe certeza de que todas las encuestas hayan sido respondidas por universitarios. Asimismo, debido a la ausencia de información sobre las variables de RE y estrategias de afrontamiento al estrés en el contexto de la pandemia por la COVID-19, podría representar una limitación porque dificultó la realización de un contraste más específico con las investigaciones antecedentes, las cuales se desarrollaron en un contexto de normalidad. Por último, es importante recalcar que a pesar de haber obtenido valores aceptables en la confiabilidad a partir de la correlación inter-ítem promedio $\left(\mathrm{r}_{\mathrm{ii}}\right)$, los valores con el coeficiente alfa fueron, en algunas ocasiones, bajos y moderados en algunas dimensiones del COPE, por lo que los análisis estadísticos pudieron verse afectados, como puede ser el análisis de regresión ya que dicho procedimiento se realiza asumiendo que las medidas obtenidas no presentan error.

En conclusión, a partir de los resultados obtenidos se ha evidenciado que la RE de los jóvenes universitarios influye de manera parcial solo en algunas de las estrategias de 
afrontamiento al estrés. Específicamente, en las estrategias del estilo centrado en el problema se encontró una influencia de la reevaluación cognitiva con el afrontamiento activo, planificación, supresión de actividades y contención de afrontamiento; mientras que la supresión solo influyó en la búsqueda de soporte social por razones instrumentales. Respecto, al estilo centrado en la emoción, no se encontró respaldo para la hipótesis planteada, pero se encontró una influencia de la reevaluación cognitiva sobre la aceptación y la reinterpretación positiva y crecimiento; para la dimensión de supresión esta presentó influencia sobre las dimensiones de búsqueda de soporte social por razones emociones, reinterpretación positiva y crecimiento. Finalmente, en el estilo evitativo, se encontró una influencia de la supresión sobre el desentendimiento conductual y la negación; la reevaluación cognitiva solo influyó sobre el uso del humor.

Se recomienda que, para futuras investigaciones, la muestra esté conformada solo por jóvenes de una universidad específica, debido a que cada institución tiene sus propios procesos ya sea en la malla curricular o el uso de las plataformas virtuales para dictar clases, dicha vivencia o experiencia educativa por parte de los universitarios podría influir en las respuestas que tengan respecto a las variables estudiadas. También se podría considerar investigar solo personas de un mismo sexo, ya sea hombres o mujeres, ya que su experiencia ante el estrés es distinta. Por otro lado, se podría abarcar un estudio haciendo una comparación entre carreras de una misma facultad, y mediante ello, obtener información más precisa de la influencia de las variables en la muestra. Asimismo, se recomienda realizar un estudio enfocado en estudiantes de los primeros ciclos de la universidad ya que recién se están adaptando al ambiente universitario o quizás en estudiantes de los últimos ciclos de la carrera, los cuales en su mayoría ya están realizando prácticas pre-profesionales y a su vez llevando cursos, ambas situaciones podrían influir en como ellos expresan sus emociones y por ende las estrategias que utilizarán. 


\section{Referencias}

American Psychological Association (2010). Principios éticos de los psicólogos y código de conducta. APA Enmienda. Recuperado de: http://www.psicologia.unam.mx/documentos/pdf/comite_etica/Codigo_APA.pdf

Ato, M., López, J., \& Benavente, A. (2013). Un sistema de clasificación de los diseños de investigación en psicología. Anales de Psicología, 29(3), 1038-1059. https://doi.org/10.6018/analesps.29.3.178511

Balluerka, N., Gómez, J., Hidalgo, D., Gorostiaga, A., Espada, J., Padilla, J. \& Santed, M. (2020). Las consecuencias psicológicas de la COVID-19 y el confinamiento. España: Editorial del País Vasco. Recuperado de: https://www.ub.edu/web/ub/ca/menu_eines/noticies/docs/Consecuencias_psicologicas_ COVID-19.pdf

Billings, A. \& Moos, R. (1984). Coping, stress, and social resources among adults with unipolar depression. Journal of Personality and Social Psychology, 46(4), 877891. https://doi.org/10.1037/0022-3514.46.4.877

Cabral, J., Matos, P., Beyers W. \& Soenens, B. (2012). Attachment, Emotion Regulation and Coping in Portuguese Emerging Adults: A Test of a Mediation Hypothesis. The Spanish $\begin{array}{llllll}\text { Journal of } & \text { Psychology, } & 15(3), & 1000 & - & 1012 .\end{array}$ https://doi.org/10.5209/rev_SJOP.2012.v15.n3.39391

Carver, C., Scheier, M. \& Weintraub, J. (1989). Assessing coping strategies: A theoretically based approach. Journal of Personality and Social Psychology, 56(2), 267-283. https://doi.org/10.1037/0022-3514.56.2.267

Carver, S. (1997). You want to measure coping but your protocol's too long: consider the brief COPE. International Journal of Behavioral Medicine, 4(1), 92-100. https://doi.org/10.1207/s15327558ijbm0401_6

Casari, L., Anglada, J. \& Daher, C. (2014) Estrategias de afrontamiento y ansiedad ante exámenes en estudiantes universitarios. Revista de Psicología, 32(2), 243-269. https://doi.org/10.18800/psico.201402.003 
Cassaretto, M. \& Chau, C. (2016). Afrontamiento al Estrés: Adaptación del Cuestionario COPE en Universitarios de Lima. Revista Iberoamericana de Diagnóstico y Evaluación, 42(2), 95 - 109. https://doi.org/10.21865/RIDEP42_95

Chau, C. \& Saravia, J. (2014). Adaptación universitaria y su relación con la salud percibida en una muestra de jóvenes de Perú. Revista Colombiana de Psicología, 23(2), 269-284. https://doi.org/10.15446/rcp.v23n2.41106

Chesney, M., Neilands, T., Chambers, D., Taylor, J. \& Folkman, S. (2006). A validity and reliability study of the coping self-efficacy scale. British Journal of Health Psychology, 11(3), 421-437. https://doi.org/10.1348/135910705X53155

Clark, L. \& Watson, D. (1995). Constructing validity: Basic issues in objective scale development. Psychological Assessment, 7(3), 309 - 319. Recuperado de: http://www.bwgriffin.com/gsu/courses/edur9131/content/Clark_validity_scaledevelop $\underline{\text { ment.pdf }}$

Córdova, J. \& Sulca, L. (2018). Estilos de afrontamiento y felicidad en estudiantes de la facultad de psicología de una universidad particular de lima. (Tesis de Licenciatura). Universidad Ricardo Palma, Lima, Perú. Recuperado de: http://repositorio.urp.edu.pe/bitstream/handle/URP/1424/TESIS\%20FINAL\%20SULC A-CORDOVA.pdf? sequence $=1 \&$ is Allowed $=\mathrm{y}$

De la Fuente, J., Amate, J., González, M., Artuch, R., García, J. \& Fadda, S. (2020). Effects of levels of self regulation and regulatory teaching on strategies of coping with academic stress in undergraduate students. Frontiers in Psychology, 11(22), 1-16. https://doi.org/10.3389/fpsyg.2020.00022

Di-Colloredo, C., Aparicio, D. \& Moreno, J. (2006). Descripción de los estilos de afrontamiento en hombres y mujeres ante la situación de desplazamiento. Psicología. Avances de la disciplina, $\quad$ 125-156. Recuperado de: https://www.redalyc.org/pdf/2972/297224996002.pdf

Faul, F., Erdfelder, E., Lang, A.. \& Buchner, A. (2007). G*Power 3: A flexible statistical power analysis program for the social, behavioral, and biomedical sciences. Behavior Research Methods, 39, 175-191. https://doi.org/10.3758/BF03193146 
Feldman, L., Gross, J., Conner, T. \& Benvenuto, M. (2001). Knowing what you're feeling and knowing what to do about it: Mapping the relation between emotion differentiation and emotion regulation. Cognition and Emotion, 15(6), 713-724. https://doi.org/10.1080/02699930143000239

Ferguson, C. (2009). An effect size primer: A guide for clinicians and researchers. Professional Psychology: Research and Practice, 40(5), 532 - 538. https://doi.org/10.1037/a0015808

Gaeta, M. \& Martín, P. (2009). Estrés y adolescencia: estrategias de afrontamiento y autorregulación en el contexto escolar. Revista de Humanidades, 15(1), 327-344. Recuperado de: https://dialnet.unirioja.es/servlet/articulo?codigo=3074506

García, R., Pérez-González, F., Pérez-Blasco, J. \& Natividad, L. (2012). Evaluación del estrés académico en estudiantes de nueva incorporación a la universidad. Revista Latinoamericana de Psicología, 44(2), 143-154. Recuperado de: https://www.redalyc.org/pdf/805/80524058011.pdf

Gargurevich, R. \& Matos, L. (2010). Propiedades psicométricas de Cuestionario de Autorregulación Emocional adaptado para el Perú (ERQP). Revista Psicología, 12, 192215. Recuperado de: https://sisbib.unmsm.edu.pe/BVRevistas/rev_psicologia_cv/v12_2010/pdf/a09.pdf

Gratz K \& Roemer L. (2004). Multidimensional assessment of emotion regulation and dysregulation: Development, factor structure, and initial validation of the difficulties in emotion regulation scale. Journal of Psychopathology and Behavioral Assessment, 26(1), 41-54. https://doi.org/10.1007/s10862-008-9102-4

Gross, J. \& Feldman, L. (2011). Emotion Generation and Emotion Regulation: One or Two Depends on Your Point of View. Emotion review: journal of the International Society for Research on Emotion, 3(1), 8-16. https://doi.org/10.1177/1754073910380974

Gross, J. \& Thompson, R. (2009). Emotion regulation: Conceptual foundations. Handbook of emotion regulation. New York, London: The Guilford Press.

Gross, J. (1999). Emotion regulation: past, present, future. Cognition and Emotion, 13(5), 551573. https://doi.org/10.1080/026999399379186 
Gross, J., \& John, O. (2003). Individual differences in two emotion regulation processes: Implications for affect, relationships, and well-being. Journal of Personality and Social Psychology, 85(2), 348-362. https://doi.org/10.1037/0022-3514.85.2.348

Gutiérrez, K. (2018). Estrategias de afrontamiento en adolescentes de quinto de secundaria de Lima Metropolitana (Tesis de Licenciatura). Universidad Ricardo Palma, Lima, Perú. Recuperado

de: https://repositorio.urp.edu.pe/bitstream/handle/URP/2462/T030_73228652_T\%20\%20 \%20GUTIERREZ\%20INGUNZA\%20KATHERINE\%20HORTENCIA.pdf?sequence $=1 \&$ isAllowed $=\mathrm{y}$

Huang, L., Xu, F. \& Liu, H. (2020). Emotional responses and coping strategies of nurses and nursing college students during COVID-19 outbreak. Nurse Education in Practice, 46(1), 1-17. https://doi.org/10.1101/2020.03.05.20031898

Lazarus, R. \& Folkman, S. (1984). Stress, Appraisal, and Coping. Nueva York: Springer.

Lazarus, R. \& Folkman, S. (1986). Estrés y procesos cognitivos. Barcelona: Martínez Roca S.A. Lévano, J. (2003). El patrón de conducta tipo A y tipo B y los estilos de afrontamiento al estrés en una muestra de personal militar. (Tesis de Maestría). Universidad Nacional Mayor de San Marcos, Lima, Perú. Recuperado de: https://sisbib.unmsm.edu.pe/bibvirtualdata/Tesis/Salud/Levano_MJ/T_completo.pdf

Lorenzo, A., Díaz, K. \& Zaldívar, D. (2020). La psicología como ciencia en el afrontamiento a la COVID-19: Apuntes generales. Ciencias sociales y humanísticas, 10(2), 1-16. Recuperado de: http://www.revistaccuba.sld.cu/index.php/revacc/article/view/839/855

Matalinares, M., Díaz, G., Arenas, C., Raymundo, O., Baca, D., Uceda, J. \& Yaringaño, J. (2016). Afrontamiento al estrés y bienestar psicológico en estudiantes universitarios de Lima y Huancayo. Revista IIPSI, 19(2), 123-143. https://doi.org/10.15381/rinvp.v19i2.12894

Midkiff, M., Lindsey, C. \& Meadows, E. (2018) The role of coping self-efficacy in emotion regulation and frequency of NSSI in young adult college students. Cogent Psychology, 5(1), 1-14. https://doi.org/10.1080/23311908.2018.1520437 
Ministerio de Salud \& Organización Panamericana de la Salud (2020). COVID-19: Respuesta de la OPS/OMS. Washington, D.C.: OPS/OMS; 2020. Recuperado de: https://iris.paho.org/handle/10665.2/52411

Monteiro, N., Balogun, S. \& Oratile, K. (2014) Managing stress: the influence of gender, age and emotion regulation on coping among university students in Botswana. International Journal of Adolescence and Youth, 19(2), 153-173. https://doi.org/10.1080/02673843.2014.908784

Muñoz, S. Molina, D., Ochoa, R., Sánchez, O. \& Esquivel, J. (2020). Estrés, respuestas emocionales, factores de riesgo, psicopatología y manejo del personal de salud durante la pandemia por COVID-19. Acta Pediátrica de México, 41(1), 127-136. http://dx.doi.org/10.18233/APM41No4S1ppS127-S1362104

Ong, E. \& Thompson, C. (2019). The importance of coping and emotion regulation in the occurrence of suicidal behavior. Psychological Reports, 122(4), 1192- 1210. https://doi.org/10.1177/0033294118781855

Ozamiz, N., Dosil, M., Picaza M., \& Idoiaga, N. (2020). Niveles de estrés, ansiedad y depresión en la primera fase del brote del COVID-19 en una muestra recogida en el norte de España. Caderno de Saúde Pública, 36(4), 1-10. https://doi.org/10.1590/0102$311 \times 00054020$

Pascual, A. \& Conejero, S. (2019). Regulación emocional y afrontamiento: Aproximación conceptual y estrategias. Revista Mexicana de Psicología, 36(1), 74-83. Recuperado de: https://www.redalyc.org/pdf/2430/243058940007.pdf

Pedró, F. (2020). COVID-19 y educación superior en América latina y el Caribe: Efectos, impacto y recomendaciones políticas. Madrid, España: Fundación Carolina. Recuperado de: https://www.fundacioncarolina.es/wp-content/uploads/2020/06/AC36.-2020.pdf

Pérez, E., \& Medrano, L. (2010). Análisis Factorial Exploratorio: Bases Conceptuales y Metodológicas. Revista Argentina de Ciencias del Comportamiento; 2(1), 58-66. Recuperado de: https://dialnet.unirioja.es/servlet/articulo?codigo=3161108 
Pérez, H., Morales, H. \& Wetzaell, M. (2002). Estilos de afrontamiento y estatus performance en un grupo de pacientes oncológicos hospitalizados. Revista de Psicología de la PUCP, 2(1), 94-131. https://doi.org/10.1590/0102-311x00054020

Pérez, I. (2014). Regulación emocional y experiencias positivas, un camino hacia la felicidad. (Proyecto final de posgrado). Universidad de Barcelona, España. Recuperado de: http://diposit.ub.edu/dspace/bitstream/2445/64104/6/TFM_Perez_Urizarbarrena.pdf

Piri, Z., Amiri, M., Bazzazian, S. \& Ghamari, M. (2019). The Mediating Role of Coping Strategies in the Relationship of Difficulties in Emotion Regulation With Internet Addiction Among College Students. Quarterly of "The Horizon of Medical Sciences", 26(1), 38-53. https://doi.org/10.32598/hms.26.1.3108

Ramón, A. \& Ramón, C. (2017). Perfiles de regulación emocional y estrés académico en estudiantes de fisioterapia. European Journal of Education and Psychology, 10(2), 5767. https://doi.org/10.1016/j.ejeps.2017.07.002

Salovey, P., Mayer, M., Goldman, S., Turvey, C. \& Palfai, T. (1995). Emotional attention, clarity, and repair: Exploring emotional intelligence using the Trait Meta-Mood Scale. In J.W. Pennebaker (Ed.), Emotion, disclosure, and health. Washington, DC: American Psychological Association. https://doi.org/10.1037/10182-006

Silva, J. (2005). Regulación emocional y psicopatología: el modelo de vulnerabilidad/ resiliencia. Revista Chilena de Neuro-Psiquiatría, 43(3), 201-209. http://dx.doi.org/10.4067/S071792272005000300004

Smrtnik, H. \& Prosen, S. (2016). Coping and emotion regulation strategies in adulthood specificities regarding age, gender and level of education. Journal for General Social Issues, 25(1), 43-62. https://doi.org/10.5559/di.25.1.03

Solís, C. \& Vidal, A. (2006). Estilos y estrategias de afrontamiento en adolescentes. Revista de Psiquiatría y Salud Mental Hermilio Valdizan, 7(1), 33-39. Recuperado de: https://www.scielo.br/scielo.php?script=sci_arttext\&pid=S1984-02922010000100003

Souto, A. (2013). Regulación emocional y estrés académico en estudiantes de fisioterapia (Tesis Doctoral). Universidad de Coruña, España. Recuperado de: https://ruc.udc.es/dspace/bitstream/handle/2183/11719/SoutoGestal_AntonioJose TD 2013.pdf? sequence $=4 \&$ is Allowed $=y$ 
The jamovi project (2020). jamovi. (Version 1.2) [Computer Software]. Recuperado de: https://www.jamovi.org

Tobin, D., Holroyd, K. \& Reynolds, R. (1984). User's manual for the coping strategies inventory. Athens, OH: Ohio University. Recuperado de: https://www.academia.edu/30133319/User_Manual_for_the_COPING_STRATEGIES _INVENTORY

Urbano, E. (2019). Estilos de afrontamiento al estrés y bienestar psicológico en adolescentes de la escuela de líderes escolares de lima norte (Tesis de maestría). Universidad Peruana Cayetano Heredia, Lima, Perú. Recuperado de: http://repositorio.upch.edu.pe/bitstream/handle/upch/6441/Estilos_UrbanoReano_Edira . pdf? sequence $=1 \&$ is Allowed $=\mathrm{y}$

Vilà Baños, R., Torrado-Fonseca, M., y Reguant-Álvarez, M. (2019). Análisis de regresión lineal múltiple con SPSS: un ejemplo práctico. REIRE Revista d'Innovació i Recerca en Educació, 12(2), 1-10. http:// doi.org/10.1344/reire2019.12.222704

Wang, C., Pan, R., Wan, X., Tan, Y., Xu, L., Ho, C. \& Ho, R. (2020). Immediate Psychological Responses and Associated Factors during the Initial Stage of the 2019 Coronavirus Disease (COVID-19) Epidemic among the General Population in China. International Journal of Environmental Research Public Health. 17(5), 1-25. https://doi.org/10.3390/ijerph17051729

Weide, J., Vicentini, E., Araujo, M., Machado, W. \& Enumo, S. (2020). Cartilla para el enfrentamiento del estrés en tiempos de pandemia. Porto Alegre, Brasil: PUC-Campinas. Trabalho. Recuperado de: https://www.puc-campinas.edu.br/wpcontent/uploads/2020/04/Cartilla-estres-esp.pdf

Wong, C., Silva, K., Kecojevic, A., Schrager, S., Jackson, J., Iverson, E. \& Lankenau, S. (2013). Coping and emotion regulation profiles as predictors of nonmedical prescription drug and illicit drug use among high-risk young adults. Drug and Alcohol Dependence. 132(0), 165-171. https://doi.org/10.1016/j.drugalcdep.2013. 01.024 
Zuckerman, M., \& Gagne, M. (2003). The COPE revised: Proposing a 5 -factor model of coping strategies. Journal of Research in Personality, 37(1), 169-204. https://doi.org/10.1016/S0092-6566(02)00563-9 


\section{Apéndice 1}

Consentimiento informado

\section{Consentimiento Informado para universitarios}

Estimado/a estudiante:

La investigación titulada "Influencia de la regulación emocional sobre las estrategias de afrontamiento ante la situación de pandemia en universitarios de Lima” es conducida por Daniela Huicho Bautista, bachiller en Psicología, perteneciente a la Universidad San Ignacio de Loyola.

Este estudio se realizará en estudiantes universitarios con la finalidad de identificar como influye la expresión de las emociones sobre las formas en las que afrontan las dificultades ante la situación de pandemia. Por ello, su participación es primordial en este proceso y estaremos agradecidos si accede.

La duración de toda la evaluación no será mayor a 10 minutos. Se solicitarán datos personales (edad, carrera, ciclo, etc.) así como la respuesta a encuestas que valoran aspectos psicológicos (p.e., estrategias de afrontamiento). La información recopilada se tratará en forma confidencial y solo se usará para fines del estudio y sin ningún otro propósito.

El proceso es completamente voluntario y puede interrumpir su participación cuando lo considere pertinente.

Si desea más información del proyecto, puede enviar un mensaje a daniela.huicho@usil.pe En ese sentido, si usted está de acuerdo en participar, puede dar click al botón SIGUIENTE. 


\section{Apéndice 2}

Ficha sociodemográfica

\section{FICHA SOCIODEMOGRÁFICA}

\begin{tabular}{|c|c|c|c|}
\hline $\begin{array}{l}\text { Carrera: } \\
\text { Universidad: } \\
\square \text { Pública } \\
\square \text { Privada } \\
\text { Sexo } \\
\square \text { Hombre } \square \text { Mujer } \\
\text { Edad__ años }\end{array}$ & $\begin{array}{l}\text { Estado Civil } \\
\square \text { Soltero(a) } \\
\square \text { Casado(a) } \\
\square \text { Divorciado(a) } \\
\square \text { Viudo(a) }\end{array}$ & $\begin{array}{l} \\
\text { Lugar de nacimiento: } \\
\square \text { Lima } \\
\square \text { Fuera de Lima }\end{array}$ & $\begin{array}{l}\text { Ciclo: } \\
\text { ¿En cuántos cursos } \\
\text { te has matriculado } \\
\text { este ciclo? }\end{array}$ \\
\hline $\begin{array}{l}\text { ¿La carrera que } \\
\text { cursas actualmente } \\
\text { siempre fue tu } \\
\text { primera opción? } \\
\text { Sí No } \\
\text { ¿Es estudiante de } \\
\text { traslado externo? } \\
\text { Sí No }\end{array}$ & $\begin{array}{l}\text { ¿La carrera que } \\
\text { cursas actualmente } \\
\text { es tu primera } \\
\text { carrera } \\
\text { profesional? } \\
\text { Sí No } \\
\text { ¿Trabajas } \\
\text { actualmente? } \\
\square \text { Sí: } \\
\square \text { No }\end{array}$ & $\begin{array}{l}\text { Considero que la } \\
\text { situación de pandemia } \\
\text { ha influido en mi } \\
\text { rendimiento: } \\
\square \text { Muy en desacuerdo } \\
\square \text { En desacuerdo } \\
\square \text { Entre uno y otro } \\
\square \text { De acuerdo } \\
\square \text { Muy de acuerdo }\end{array}$ & $\begin{array}{l}\text { ¿Cómo ha sido tu } \\
\text { rendimiento en este } \\
\text { último ciclo? } \\
\square \text { Muy bueno } \\
\square \text { Bueno } \\
\square \text { Regular } \\
\square \text { Malo } \\
\square \text { Muy malo }\end{array}$ \\
\hline
\end{tabular}

